International Journal of Engineering \& Technology, $7(4)(2018) 2013-2027$
International Journal of Engineering \& Technology
SPC
Website: www.sciencepubco.com/index.php/IJET
doi: $10.14419 /$ ijet. $v 74$ it.14116
Research paper

\title{
Chemical, thermal and economic aspects for the energy balance of coal gasification power plants with and without $\mathrm{CO}_{2}$ recovery
}

\author{
Said M. A. Ibrahim ${ }^{1}$ *, Mostafa E. M. Samy ${ }^{1}$ \\ ${ }^{1}$ Department of Mechanical Engineering, Al-Azhar University, Nasr City, Cairo 11371, Egypt \\ *Corresponding author E-mail: prof.dr.said@hotmail.com
}

\begin{abstract}
This paper presents a methodology for studying the chemical, thermal processes and economics for the gasification systems employed in coal fired power stations through thermodynamic analyses based on thermodynamic laws in order to gain some new aspects of the plant performance. A resourceful computer program is developed and designed to calculate all necessary design and performance data for four selected coal fired power plants for all coal ranks. Detailed manual calculations were performed for the results. Comparison of computer and manual results are in excellent agreement which indicates that the present program is an accurate quick powerful tool for all users. The main findings of this paper are that Integrated Coal Gasification Combined Power Generation Plants with $\mathrm{CO}_{2}$ recovery increase the plant's thermal efficiency and decrease the $\mathrm{CO}_{2}$ emission. The thermodynamics, hydrodynamics, and kinetics of each reaction to the gas combinations were most likely tested by each of the techniques when using a variety of fuels under the condition of "Oxygen gas at 30 bar pressure". The economic analysis is based on analyzing the economics of carbon dioxide capture and storage and the amount of carbon dioxide emitted from power plants. Finally, with new developments, the capture and sequestration of $\mathrm{CO}_{2}$ could lead to.
\end{abstract}

Keywords: Coal Gasification and Syngas; Efficiency; Power Plant; $\mathrm{CO}_{2}$ Recovery; Cost.

\section{Introduction}

IGCC is an acronym for Integrated Gasification Combined Cycle. The main purpose of IGCC is to use hydrocarbon fuels in solid or liquid forms to produce cleaner and more efficient electric power via gasification compared to the direct combustion of fuel. Hydrocarbon fuels can include coal, biomass, refinery bottom residues, and municipal wastes. The way to produce a cleaner power is firstly to convert solid/liquid fuels to gas, so that they can be cleaned before being burned by getting rid mainly of particulates, sulfur, mercury, and other trace elements. The cleaned gas, called synthesis gas (syngas), consists primarily of carbon monoxide and hydrogen , can then be fed to a conventional combined cycle to produce electricity [1].

Thermal integration can be incorporated by interconnecting the different grades of steam generated during the cooling of the syngas, gas cleanup, and/or water gas shift processes with the heat recovery steam generator (HRSG) and the steam turbine. Full air integration will enhance the overall plant efficiency by approximately 3 to $4 \%$. However, this increases the complexity of construction, operation, and maintenance, which may result in increasing the construction phase delay and/or cost overrun, increased maintenance, low availability, and degraded reliability. Accordingly, the concept of nonintegrated IGCC has been raised by some developers to trade reduced efficiency for higher availability and reliability, even though the term "nonintegrated IGCC" could be confusing [1].

When global warming became a serious concern, the emission of carbon dioxide a greenhouse gas (GHG) from power stations was subjected to stringent regulations. $\mathrm{CO}_{2}$ is produced profusely and becomes the main culprit in global warming. Practically, there are three methods to reduce $\mathrm{CO}_{2}$ emissions: increasing the plant's overall efficiency, capturing a portion of $\mathrm{CO}_{2}$ and sequestering it, which is known as carbon capture and sequestration (CCS), or utilizing the captured $\mathrm{CO}_{2}$ several times. The syngas generated by means of the gasification process can be readily separated into highly concentrated $\mathrm{H}_{2}$ and $\mathrm{CO}_{2}$ through the water-gas shift (WGS) process before the combustion stage (i.e., pre combustion) in an IGCC plant, as opposed to pulverized coal (PC) power stations, which have to use a post-combustion carbon capture method. It is significantly cheaper to perform pre combustion carbon capture in an IGCC system rather than post-combustion carbon capture in a PC power plant due to the nature of the processes involved and the reduced size of equipment. CCS exerts a severe penalty on power output, plant efficiency, and cost of electricity (COE) [1].

\section{Computer program}

A main aim of this research is to develop and design a commercial useful computer program to calculate all the necessary data for the different coal power plants for all ranks of coal.. It is a quick resourceful powerful easy tool for getting all the necessary chemical, thermodynamic, and energy calculations in addition to cost of electricity from any plant. The output results are many as they are proportional to the complexity of the program. Four computer programs were designed; one for each of the studied power stations.

The present program is a simulation of several power plants including four main types, power plants without coal gasification 
with $\mathrm{CO}_{2}$ recovery and storage, and power plants with coal gasification and $\mathrm{CO}_{2}$ recovery and storage.

Each program consists of input, process, control, and output. The input data which provide all output data include: mass flow rate of coal, efficiency of combustor and so on. This includes many processes in the cycle which represent chemical, heat transfer, accountant, and economic processes. The economic process as one of the main results of the programs includes all cycle costs as represented by capital cost, operating cost, $\mathrm{COE}$, and cost of $\mathrm{CO}_{2}$ capture analogue to plant, cost of $\mathrm{CO}_{2}$ with capture avoided, and so on. The control in the program is established to change the state according to coal type which depends on the heating value of coal, $\mathrm{CO}_{2}$ capture and storage system which reflects on the efficiency of $\mathrm{CO}_{2}$ capture, the high pressure (HP) $\mathrm{CO}_{2}$ capture which reflects on the efficiency of $\mathrm{CO}_{2}$ compressor.

In the first program used for steam power plants the input data are mass flow rate of coal, high heating value (HHV) of coal; efficiencies of: boiler, heat transfer of boiler, steam turbine, condenser, $\mathrm{CO}_{2}$ compressor, and $\mathrm{CO}_{2}$ capture; enthalpies of: water inlet to boiler, steam outlet from boiler or steam inlet to turbine, steam outlet from turbine, $\mathrm{CO}_{2}$ inlet to compressor at $1 \mathrm{bar}$, and $\mathrm{CO}_{2}$ outlet from compressor at 150 bar; capital cost of plant, coal cost, and life time of plant.

In the second program for entrained flow gasification power plants the input data are mass flow rate of coal, HHV of coal; efficiencies of: syngas cooler, $\mathrm{CO}_{2}$ capture, gas turbine, air compressor, HRGS, $\mathrm{CO}_{2}$ compressor, steam turbine, condenser, air compressor, and ASU; enthalpies of: water inlet to syngas cooler or water inlet to HRGS or condenser cooling water or cooling water inlet for $\mathrm{CO}_{2}$ capture, steam outlet from syngas cooler or steam outlet from HRGS steam inlet to turbine), air inlet to compressor or air inlet to compressor of ASU, air outlet from compressor, gas inlet to turbine, gas outlet from turbine or gas inlet to HRGS, gas outlet from HRGS , $\mathrm{CO}_{2}$ inlet to compressor at $1 \mathrm{bar}, \mathrm{CO}_{2}$ inlet to compressor at 15.8 bar, $\mathrm{CO}_{2}$ outlet from compressor at 150 bar, steam outlet from HRGS for $\mathrm{CO}_{2}$ capture, steam outlet from turbine, and air outlet from compressor of ASU; in addition to same cost items as before.

In the programs used for fluidized bed gasification and fixed bed gasification power plants the input data are as in the second program in addition to enthalpy of steam outlet from HRGS to gasifier.

When discussing the control of the program, reference should be made to the data on which the control steps are based on in the program. These data are similar in all the programs of the studied power stations, and are: (1) coal HHV which controls the program by determining the type of coal that is used in the plant, and the program is run according to the corresponding HHV of the used coal $(=33000,23600,16800,27800 \mathrm{~kJ} / \mathrm{kg}$ for bituminous, subbituminous, lignite, and anthracite coals, respectively), (2) efficiency of $\mathrm{CO}_{2}$ capture; this controls the program by determining whether the station is operating a $\mathrm{CO}_{2}$ recovery system or not. For a system without $\mathrm{CO}_{2}$ recovery, the system efficiency is zero, and if employing $\mathrm{CO}_{2}$ recovery then the system efficiency is according to the operating conditions and so on, and (3) efficiency of $\mathrm{CO}_{2}$ compressor which controls the program to specify whether the station is operating a high-pressure carbon dioxide recovery system or not. If not then the compressor efficiency is zero, and if used then the pressure of $\mathrm{CO}_{2}$ is increased according to compressor efficiency and so on.

In the program for steam power plants the output data are mass flow rates of: air inlet for complete combustion, outlet steam from boiler, and cooling water in condenser, and $\mathrm{CO}_{2}$ emission; mass of $\mathrm{CO}_{2}$ captured, molar weight of coal, thermal cycle efficiency, heat of combustion in boiler, power of steam turbine, power of $\mathrm{CO}_{2}$ compressor, output power of cycle, total cost for useful life of plant, total cost in first year, operating costs, energy output, and COE.

For entrained flow, fluidized bed, and fixed bed gasification power plants the output data are mass flow rates of: carbon, $\mathrm{H}_{2}, \mathrm{O}_{2}$, $\mathrm{N}_{2}, \mathrm{~S}$, moisture, and ash in coal, oxygen outlet from ASU, air in
ASU, $\mathrm{N}_{2}$ outlet from ASU, water in slurry, water in slag, $\mathrm{H}_{2}$ in syngas, $\mathrm{CO}$ in syngas, $\mathrm{CH}_{4}$ in syngas, $\mathrm{CO}_{2}$ in syngas, $\mathrm{N}_{2}$ in syngas, steam in syngas, $\mathrm{H}_{2} \mathrm{~S}$ in syngas, syngas, outlet steam from syngas cooler, air inlet for complete combustion, syngas after cleaning, air inlet to compressor, nitrogen inlet for combustion, inlet gas to turbine, steam outlet steam to HRGS, total steam inlet to turbine, cooling water, $\mathrm{CO}_{2}$ emission for complete combustion, steam required for $\mathrm{CO}_{2}$ capture, $\mathrm{CO}_{2}$ captured, $\mathrm{H}_{2}$ after $\mathrm{CO}_{2}$ capture, $\mathrm{CO}$ after $\mathrm{CO}_{2}$ capture, and $\mathrm{CO}_{2}$ emissions; molar weight of coal, heat of combustion of syngas, summation of heat in syngas cooler, heat of combustor, heat of combustion of syngas after $\mathrm{CO}_{2}$ capture, heat released by carbon capture, cold gas efficiency, carbon conversion efficiency, gas turbine efficiency, mass of $\mathrm{CO}_{2}$ emissions, thermal cycle efficiency, power of air compressor, power of gas turbine, net power of gas turbine, power of steam turbine, power of $\mathrm{CO}_{2}$ compressor, cycle output power, power of air compressor of ASU, total cost for useful life time of plant, total cost in first year, operating costs, energy output and COE.

The present programs provide: (1) the capacity, efficiency and economics of the plant, whether coal gasification includes recovery of carbon dioxide and storage or without gasification or retrieval at different situations and these cases include: climatic, economic, strategic, and political situations, (2) make comparisons of results between plants, whether in terms of efficiency or cost per station and to give important guidance when operating the station, and (3) offer comparisons of the results between all stations to determine the best of them.

\section{Methodology}

Step by step manual calculations are conducted for the thermal and energy balances with a comprehensive cost model for each of the selected power plants. These stations include coal-fired ones with and without the possibility of recovering carbon dioxide, as well as stations without coal gasification with and without $\mathrm{CO}_{2}$ recovery.These results are then compared with those obtained from the present computer programs. The following procedure indicates the calculation processes in the present programs.

\subsection{Reference calculation data}

The following manual calculations are made for only one type of coal, bituminous, based on the data provided in Table 1, for all the studied power stations. The present computer program can provide such results and more for all coal ranks.

Table 1: Design Technical Data

\begin{tabular}{ll}
\hline Coal type (Bituminous) & \\
\hline Mass of coal & $2500 \mathrm{TPD}$ \\
Gasifier pressure & $30 \mathrm{bar}$ \\
Steam pressure & $9 \mathrm{MPa}$ \\
Steam temperature & $1150^{\circ} \mathrm{C}$ \\
Pressure ratio of gas turbine & 15.8 \\
Air temperature & $27^{\circ} \mathrm{C}$ \\
Water temperature & $25^{\circ} \mathrm{C}$ \\
Condensate pressure & $0.1 \mathrm{bar}$ \\
Efficiency of any system & $100 \%$ \\
Any losses & $0 \%$ \\
\hline
\end{tabular}

\section{- Carbon conversion}

Table 2 gives carbon conversion ratios (CCR) or carbon conversion efficiency (CCE) for different gasifiers, as taken from Ref. [1]. These represent the mass percentage of total carbon in the gasifier feedstock (i.e., coal or biomass) which is converted to syngas for different gasification technologies.

The values in Table 2 are used in the next thermodynamic calculations for power plants, since the calculations depend on them.

Table 2: Carbon Conversion

\begin{tabular}{ll}
\hline Gasifier & Carbon conversion \% \\
\hline Entrained flow & 99.999 \\
Fluidized bed & 97 \\
\hline
\end{tabular}




\section{Fixed/moving bed} 98

The entrained flow gasifier exhibits high carbon conversion because of the high temperature involved and hence low tar. The fluidized bed gasifier has the lowest carbon conversion because the solid waste agglomerates and results in high tar.

\subsection{Algorithm procedure}

1) Calculate the thermodynamic properties (pressure $P$, temperature $\mathrm{T}$, entropy s, enthalpy $\mathrm{h}$, moisture content $\mathrm{X}$; at inlet and outlet in all parts of the plant's components).

2) Perform heat balance for feed water, and generated steam and gases.

3) Calculate the useful work of the turbines.

4) Calculate the amount of heat added to generate steam by syngas cooler and heat recovery gas system as well as the amount of heat rejected from the condenser and calculate the efficiency of the plant.

5) Determine all chemical reactions in the gasifier and carbon capture system and determine the heat from these reactions.

6) Determine the economics of the plant.

\subsection{Thermodynamic equations}

- $\quad$ Boiler in steam power plant

Total heat in boiler $=\dot{\mathrm{m}}_{\mathrm{C}} \times \mathrm{HHV}=\dot{\mathrm{m}}_{\mathrm{st}}, \mathrm{w}\left(\mathrm{h}_{\text {out }}-\mathrm{h}_{\text {in }}\right)$

Where

$\dot{\mathrm{m}}_{\mathrm{C}}=$ mass flow rate of coal, tone/day $(\mathrm{TPD})$,

$\mathrm{HHV}=$ high heating value of coal, $\mathrm{kJ} / \mathrm{kg}$,

$\dot{\mathrm{m}} \mathrm{St}, \mathrm{w}=$ mass flow rate of steam or water, TPD,

$\mathrm{h}_{\text {out }}=$ enthalpy of outlet steam, $\mathrm{kJ} / \mathrm{kg}$. and

$\mathrm{h}_{\text {in }}=$ enthalpy of inlet water, $\mathrm{kJ} / \mathrm{kg}$.

- $\quad$ Syngas cooler in IGCC power plant

Total heat in syngas cooler $=\left[\mathrm{CCE} \times\left(\dot{\mathrm{m}}_{\mathrm{C}} \times \mathrm{HHV}\right)\right]-\Delta \mathrm{HC}=\dot{\mathrm{m}}_{\mathrm{st}}, \mathrm{w}$

(hout $-h_{\text {in }}$

Where

$\mathrm{CCE}=$ carbon conversion efficiency, \%,

$\dot{\mathrm{m}}_{\mathrm{C}}=$ mass flow rate of coal, $\mathrm{kg} / \mathrm{s}$,

$\mathrm{HHV}=$ high heating value of coal, $\mathrm{kJ} / \mathrm{kg}$

$\Delta \mathrm{H}_{\mathrm{C}}=$ heat of combustion of product gases from gasifier, $\mathrm{kW}$,

$\dot{\mathrm{m}}_{\mathrm{St}, \mathrm{w}}=$ mass flow rate of steam or water, $\mathrm{kg} / \mathrm{s}$,

$\mathrm{h}_{\text {out }}=$ enthalpy of outlet steam, $\mathrm{kJ} / \mathrm{kg}$, and

$\mathrm{h}_{\mathrm{in}}=$ enthalpy of inlet water, $\mathrm{kJ} / \mathrm{kg}$.

- Carbon capture (pre combustion) system in IGCC

$\Delta \mathrm{H}=\Delta \mathrm{H}$ without ccs $-\Delta \mathrm{H}$ with ccs $=\dot{\mathrm{m}}_{\mathrm{w}}\left(\mathrm{h}_{\mathrm{o}}-\mathrm{h}_{\text {in }}\right)$

where

$\Delta \mathrm{H}=$ heat rejected from CCS, $\mathrm{kW}$,

$\Delta \mathrm{H}_{\text {without ccs }}=$ heat of combustion of syngas before CCS, $\mathrm{kW}$,

$\Delta \mathrm{H}$ with ccs $=$ heat of combustion of syngas after CCS, $\mathrm{kW}$,

$\dot{\mathrm{m}}_{\mathrm{w}}=$ mass flow rate of water, $\mathrm{kJ} / \mathrm{kg}$,

$\mathrm{h}_{\mathrm{o}}=$ enthalpy of outlet water, $\mathrm{kJ} / \mathrm{kg}$, and

$\mathrm{h}_{\text {in }}=$ enthalpy of inlet water, $\mathrm{kJ} / \mathrm{kg}$.

\section{- Gas turbine}

$\Delta \mathrm{H}_{\mathrm{C}}=\mathrm{h}_{3}\left(\dot{\mathrm{m}}_{\mathrm{air}}+\dot{\mathrm{m}}_{\mathrm{N} 2}+\dot{\mathrm{m}}_{\mathrm{Syn}}\right)-\mathrm{h}_{2} \dot{\mathrm{m}}_{\mathrm{ai}}$

$\dot{\mathrm{m}}_{\mathrm{gas}}=\dot{\mathrm{m}}_{\text {air }}+\dot{\mathrm{m}}_{\mathrm{N} 2}+\dot{\mathrm{m}}_{\mathrm{Syn}}$

$\mathrm{P}_{\mathrm{C}}=\dot{\mathrm{m}}_{\text {air }}\left(\mathrm{h}_{2}-\mathrm{h}_{1}\right)$

$\mathrm{P}_{\mathrm{T}}=\dot{\mathrm{m}}_{\text {gas }} \times\left(\mathrm{h}_{3}-\mathrm{h}_{4}\right)$

$\mathrm{W}_{\text {net }}=\mathrm{PT}-\mathrm{PC}$

$\eta_{C}=\left[\mathrm{W}_{\text {net }} / \Delta \mathrm{H}_{\mathrm{C}}\right] \times 100$
Where

$\Delta \mathrm{H}_{\mathrm{C}}=$ heat of combustor, $\mathrm{kW}$

$\dot{\mathrm{m}}_{\mathrm{air}}=$ mass flow rate of air, $\mathrm{kg} / \mathrm{s}$,

$\dot{\mathrm{m}}_{\mathrm{N} 2}=$ mass flow rate of $\mathrm{N}_{2}, \mathrm{~kg} / \mathrm{s}$,

$\dot{\mathrm{m}}_{\mathrm{syn}}=$ mass flow rate of syngas, $\mathrm{kg} / \mathrm{s}$,

$\dot{\mathrm{m}}_{\mathrm{gas}}=$ mass flow rate of syngas, $\mathrm{kg} / \mathrm{s}$,

$\mathrm{P}_{\mathrm{C}}=$ power of compressor, $\mathrm{kW}$,

$\mathrm{h}_{1}=$ enthalpy of air inlet to compressor, $\mathrm{kJ} / \mathrm{kg}$,

$\mathrm{h}_{2}=$ enthalpy of air outlet from compressor, $\mathrm{kJ} / \mathrm{kg}$

$\mathrm{P}_{\mathrm{T}}=$ power of turbine, $\mathrm{kW}$,

$\mathrm{h}_{3}=$ enthalpy of air inlet to compressor, $\mathrm{kJ} / \mathrm{kg}$,

$\mathrm{h}_{4}=$ enthalpy of air outlet from compressor, $\mathrm{kJ} / \mathrm{kg}$

$\mathrm{W}_{\text {net }}=$ net work of gas turbine, $\mathrm{kW}$, and

$\eta_{\mathrm{C}}=$ efficiency of gas turbine, $\%$.

- Heat recovery in gas system

$\dot{\mathrm{m}}_{\mathrm{w}} \mathrm{h}_{\mathrm{w}}+\dot{\mathrm{m}}_{\mathrm{G} 1} \mathrm{~h}_{\mathrm{G} 1}+\dot{\mathrm{m}}_{\mathrm{m} 1} \mathrm{~h}_{\mathrm{m} 1}=\dot{\mathrm{m}}_{\mathrm{st}} \mathrm{h}_{\mathrm{st}}+\dot{\mathrm{m}}_{\mathrm{G} 2} \mathrm{~h}_{\mathrm{G} 2}+\dot{\mathrm{m}}_{\mathrm{m} 2} \mathrm{~h}_{\mathrm{m} 2}$

Where

$\dot{\mathrm{m}}_{\mathrm{w}}=$ mass flow rate of inlet water, TPD,

$\mathrm{h}_{\mathrm{w}}=$ enthalpy of inlet water, $\mathrm{kJ} / \mathrm{kg}$,

$\dot{\mathrm{m}}_{\mathrm{G} 1}=$ mass flow rate of inlet gas, TPD,

$\mathrm{h}_{\mathrm{G} 1}=$ enthalpy of inlet gas, $\mathrm{kJ} / \mathrm{kg}$,

$\dot{\mathrm{m}}_{\mathrm{ml}}=$ mass flow rate of steam mixture from CCS, TPD,

$\mathrm{h}_{\mathrm{ml}}=$ enthalpy of steam mixture from CCS, $\mathrm{kJ} / \mathrm{kg}$,

$\dot{\mathrm{m}}_{\mathrm{st}}=$ mass flow rate of steam inlet to turbine, TPD,

$\mathrm{h}_{\mathrm{st}}=$ enthalpy of steam inlet to turbine, $\mathrm{kJ} / \mathrm{kg}$,

$\dot{\mathrm{m}}_{\mathrm{G} 2}=$ mass flow rate of outlet gas, TPD,

$\mathrm{h}_{\mathrm{G} 2}=$ enthalpy of outlet gas, $\mathrm{kJ} / \mathrm{kg}$,

$\dot{\mathrm{m}}_{\mathrm{m} 2}=$ mass flow rate of inlet steam CCS, TPD, and

$\mathrm{h}_{\mathrm{m} 2}=$ enthalpy of inlet steam CCS, $\mathrm{kJ} / \mathrm{kg}$.

- Steam turbine

$\mathrm{P}_{\mathrm{T}}=\dot{\mathrm{m}}_{\mathrm{st}} \times\left(\mathrm{h}_{\mathrm{in}}-\mathrm{h}_{\mathrm{o}}\right)$

Where

$\mathrm{P}_{\mathrm{T}}=$ power of steam turbine, $\mathrm{kW}$,

$\dot{\mathrm{m}}_{\mathrm{st}}=$ mass flow rate of inlet steam, $\mathrm{kg} / \mathrm{s}$,

$\mathrm{h}_{\text {in }}=$ enthalpy of inlet steam, $\mathrm{kJ} / \mathrm{kg}$, and

$\mathrm{h}_{\mathrm{o}}=$ enthalpy of outlet steam, $\mathrm{kJ} / \mathrm{kg}$.

- Condenser

$\dot{\mathrm{m}}_{\mathrm{st}}\left(\mathrm{h}_{\mathrm{in}}-\mathrm{h}_{\mathrm{o}}\right)_{\mathrm{st}}=\dot{\mathrm{m}}_{\mathrm{w}}\left(\mathrm{h}_{\mathrm{o}}-\mathrm{h}_{\text {in }}\right)_{\mathrm{w}}$

Where

$\dot{\mathrm{m}}_{\mathrm{st}}=$ mass flow rate of inlet steam inlet, TPD,

$\left(h_{\text {in }}-h_{o}\right)_{s t}=$ enthalpy of inlet and outlet steam, $\mathrm{kJ} / \mathrm{kg}$,

$\dot{\mathrm{m}}_{\mathrm{w}}=$ mass flow rate of inlet water, TPD, and

$\left(\mathrm{h}_{\mathrm{o}}-\mathrm{h}_{\mathrm{in}}\right)_{\mathrm{w}}=$ enthalpy of water outlet and inlet, $\mathrm{kJ} / \mathrm{kg}$.

- Oxygen blown in gasifier (main compressor)

$\mathrm{P}_{\mathrm{C}}=\dot{\mathrm{m}}_{\text {air }}\left(\mathrm{h}_{\mathrm{o}}-\mathrm{h}_{\text {in }}\right)$

Where

$\mathrm{P}_{\mathrm{C}}=$ power of main compressor, $\mathrm{kW}$,

$\dot{\mathrm{m}}_{\text {air }}=$ mass flow rate of air, $\mathrm{kg} / \mathrm{s}$,

$\mathrm{h}_{\mathrm{o}}=$ enthalpy of outlet air, $\mathrm{kJ} / \mathrm{kg}$, and

$\mathrm{h}_{\text {in }}=$ enthalpy of inlet air, $\mathrm{kJ} / \mathrm{kg}$.

\subsection{Energy balance and economics of steam power plant without CCS}

The chosen steam power plant, depicted in Fig. 1 contains the boiler, heater tubes, boiler drum, steam turbines, generator, cooling tower, pumps, valves and condenser. The output of this station is $475 \mathrm{MW}$ and its thermal efficiency is $49 \%$ with a capital cost of $\$ 520$ million. 


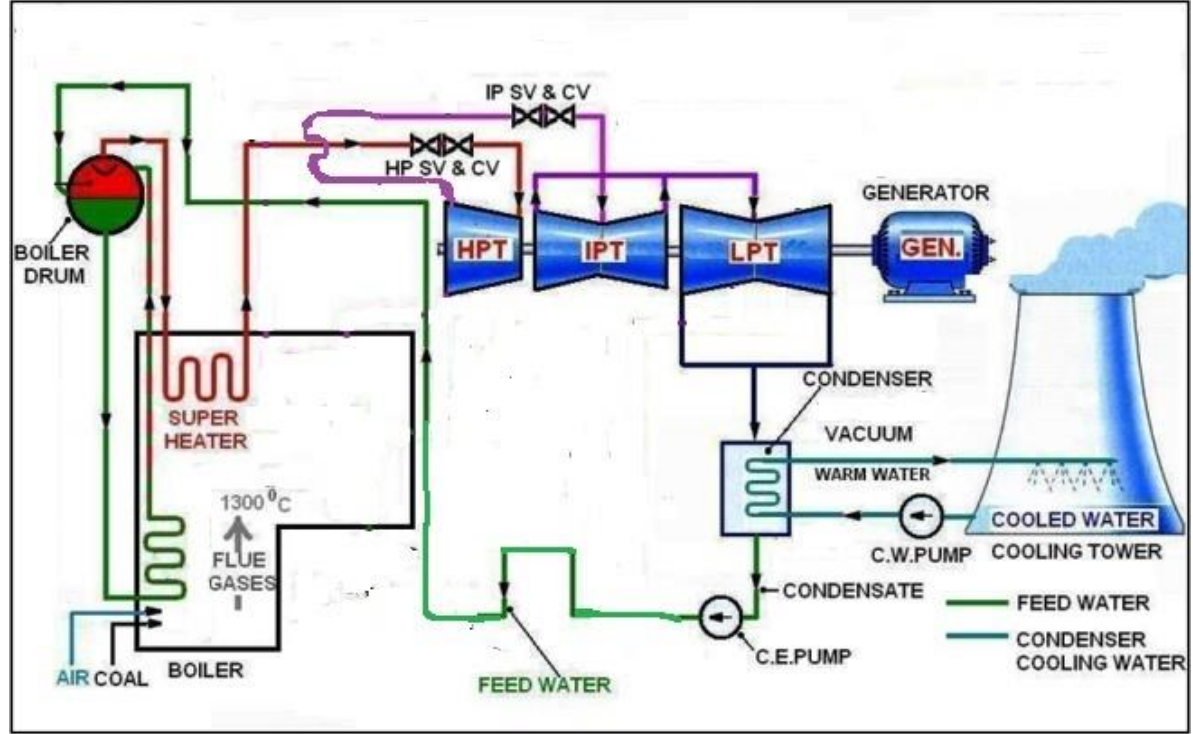

Fig. 1: Steam Power Plant without CCS [2].

- Properties of coal

Coal type: Bituminous coal

Coal composition: $\mathrm{C}_{0.67} \mathrm{O}_{0.022} \mathrm{~N}_{0.0116} \mathrm{~S}_{0.008}$

$\mathrm{M}_{\mathrm{C}}=100 / 6.725=14.869 \mathrm{gm} / \mathrm{mole}$

Moisture $=3.3 \%$ of weight

Ash $=6.2 \%$ of weight

$\dot{\mathrm{m}}_{\mathrm{C}}=2500 \mathrm{TPD} \mathrm{HHV}=33.3 \mathrm{MJ} / \mathrm{kg}$

- Boiler

- Chemical reaction in boiler

$\mathrm{C} \mathrm{H}_{0.67} \mathrm{O}_{0.022} \mathrm{~N}_{0.0116} \mathrm{~S}_{0.008}+1.1645\left(\mathrm{O}_{2}+3.78 \mathrm{~N}_{2}\right)=\mathrm{CO}_{2}+0.335$

$\mathrm{H}_{2} \mathrm{O}+0.008 \mathrm{SO}_{2}+4.40181 \mathrm{~N}_{2}$

$\begin{array}{llllll}14.869 & 160.51468 & 44 & 6.03 & 0.512 & 123.25068\end{array}$

2500TPD $26988.147397 .942 \quad 1013.8543 \quad 86.08514420722 .759$

For complete combustion, ma $=26988.143 \mathrm{TPD}=312.363 \mathrm{~kg} / \mathrm{s}$

- Calculations of boiler

Summation of heat in boiler, $\mathrm{S}=[\dot{\mathrm{m}} \times \mathrm{HHV}]$ coal

$\mathrm{S}=963541.67 \mathrm{~kW}$

This heat converts water to superheated steam

Steam: $\mathrm{P}=9 \mathrm{MPa} \mathrm{T}=1150^{\circ} \mathrm{C} \mathrm{h}=4997 \mathrm{~kJ} / \mathrm{kg}$

Water: $\mathrm{P}=9 \mathrm{MPa} \mathrm{T}=25{ }^{\circ} \mathrm{C} \mathrm{h}=105 \mathrm{~kJ} / \mathrm{kg}$

$\dot{\mathrm{m} S t} \times 4892=963541.67$

$\dot{\mathrm{m}}_{\mathrm{St}}=196.962 \mathrm{~kg} / \mathrm{s}=17017.517 \mathrm{TPD}$

- $\quad$ Steam turbine

1) Inlet conditions

$\dot{\mathrm{m}}_{\text {st in }}=196.962 \mathrm{~kg} / \mathrm{s}, \mathrm{T}=1150^{\circ} \mathrm{C}, \mathrm{P}=9 \mathrm{MPa}$,

$\mathrm{h}=4997 \mathrm{~kJ} / \mathrm{kg}, \mathrm{s}=8.1648 \mathrm{~kJ} / \mathrm{kg} \mathrm{K}$

2) Outlet conditions

$\mathrm{T}=45^{\circ} \mathrm{C}, \mathrm{P}=0.1 \mathrm{bar}, \mathrm{X}=1, \mathrm{~h}=2584.7 \mathrm{~kJ} / \mathrm{kg}, \mathrm{s}=8.1648 \mathrm{~kJ} / \mathrm{kg} \mathrm{K}$

Power of turbine $=\dot{\mathrm{mst}} \times\left(\mathrm{h}_{\mathrm{in}}-\mathrm{h}_{\mathrm{o}}\right)=475131.43 \mathrm{~kW}$

- Condenser

The cooling water cools steam in the condenser from saturated steam $X=1$ to saturated water $X=0$; this cooling water is coming from a cooling tower in which the inlet temperature $=50{ }^{\circ} \mathrm{C}$ and the temperature outlet $=25{ }^{\circ} \mathrm{C}$. So one can get the mass flow rate of cooling water, $\dot{\mathrm{m}}_{\mathrm{w}}$ from the energy balance equation

$\dot{\mathrm{m}}_{\mathrm{St}} \times\left(\mathrm{h}_{\mathrm{in}}-\mathrm{h}_{\mathrm{o}}\right)=\dot{\mathrm{m}}_{\mathrm{w}} \times\left(\mathrm{h}_{\mathrm{o}}-\mathrm{h}_{\mathrm{in}}\right)$

$196.962 \times(2584.7-105)=\dot{\mathrm{m}}_{\mathrm{w}} \times 4.18 \times(50-25)$

Mass of cooling water $=4673.7481 \mathrm{~kg} / \mathrm{s}=403811.83 \mathrm{TPD}$

- Cycle efficiency

Total power $=475131.43 \mathrm{~kW}$ $\eta_{\text {th }}=\left[\mathrm{P}_{\text {Total }} / \dot{\mathrm{m}}_{\mathrm{C}} \mathrm{HHV}\right] \times 100=49.311 \%$

- $\mathrm{CO}_{2}$ Emission

Mass of $\mathrm{CO}_{2}$ emission $=7397.942 \mathrm{TPD}$

$\mathrm{CO}_{2}$ emission $\%=100 \%$

- Economics

a) Capital cost per unit power output $=1095 \$ / \mathrm{kW}$

Capital cost $=\$ 520.271$ million

Output power $=475131.43 \mathrm{~kW}$

b) The cost of electricity (COE) per unit energy output $(\$ / \mathrm{kWh})$.

The COE is calculated over the entire useable life of the plant.

The entire useable life of the plant $=20$ years

Capital cost $=\$ 520.271$ million

Operating costs $=$ ??

- Fuel $=$ Bituminous coal

Tone $=\$ 47$

$\dot{\mathrm{m}}_{\text {Coal }}=2500 \mathrm{TPD}$

Fuel cost $=2500 \times 47 \times 365=42887500 \$ /$ year

- Operation and maintenance $(\mathrm{O} \& \mathrm{M})$ cost $=2 \%$ of the capital cost / year

$\mathrm{O} \& \mathrm{M}$ cost $=0.02 \times 520.271=10.405 \$ \mathrm{M} /$ year

So the operating costs $=53.292 \$ \mathrm{M} /$ year

For 20 years the costs $=520.271+(53.292 \times 20)=\$ 1586.111$ million

Unit output energy $=(475131.43 \times 8760) / 1000=4162151.3$ MWh

Total cost in first year $\$$ million $=\$ 573.563$ million

Cost of electricity $(\mathrm{COE})$ per unit energy output $=137.804$ $\$ / \mathrm{MWh}$

\subsection{Energy balance and economics of entrained flow gasification plant without CCS.}

In Fig. 2 the selected entrained flow gasification plant is shown which contains the gasifier, heater tubes, syngas cooler, gas cleanup, air separation unit, slurry plant, gas turbine, steam turbine, generators, cooling tower, pumps, valves and condenser. The plant produces $593 \mathrm{MW}$ and $61.5 \%$ efficiency and a capital cost of \$ 678.7 million. 


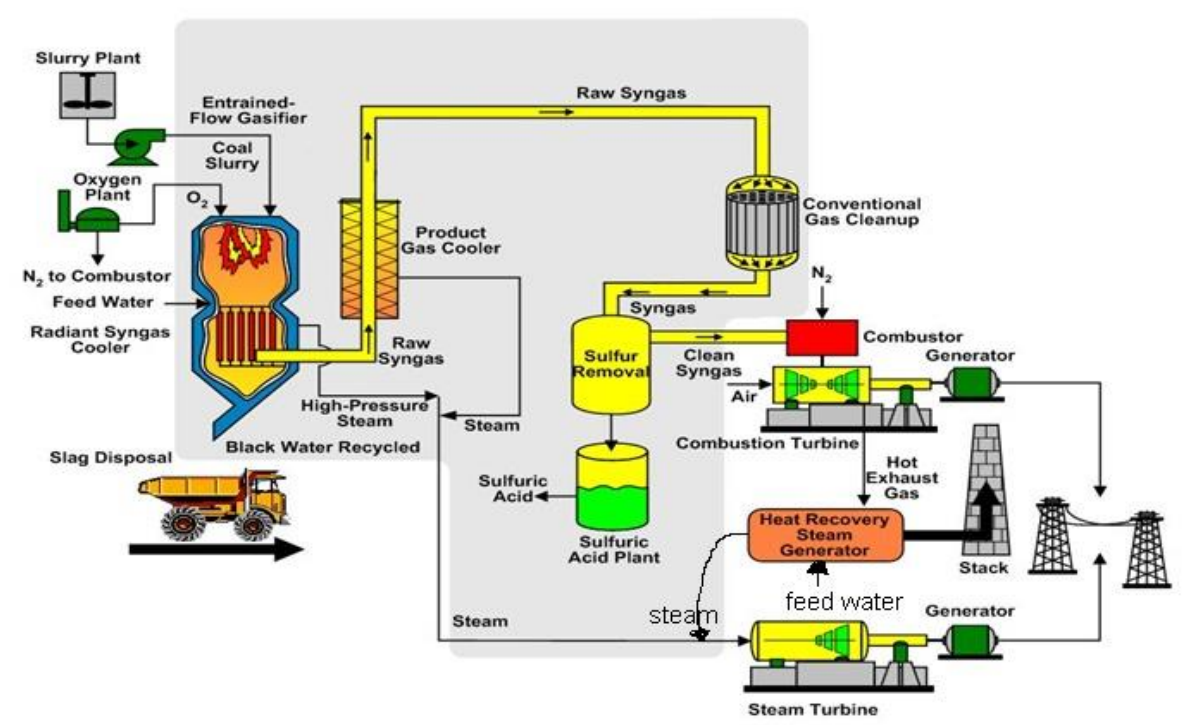

Fig. 2: Entrained Flow Gasification Plant without CCS [3].

- Properties of coal

Coal type: Bituminous coal

Coal composition: $\mathrm{C} \mathrm{H}_{0.67} \mathrm{O}_{0.022} \mathrm{~N}_{0.0116} \mathrm{~S}_{0.008}$

$\mathrm{M}_{\mathrm{C}}=100 / 6.725=14.869 \mathrm{gm} / \mathrm{mole}$

Moisture $=3.3 \%$ of weight

Ash $=6.2 \%$ of weight

$\dot{\mathrm{m}}_{\mathrm{Coal}}=2500 \mathrm{TPD}$

mo2 $=2100$ TPD $84 \%$ by weight of coal

$\dot{\mathrm{m}}_{\mathrm{H} 2 \mathrm{O}}=1008.81 \mathrm{TPD} 40.352 \%$ of [slurry feed]

Moisture $=82.5$ TPD Ash $=155$ TPD

- Gasifier

- Chemical reaction in gasifier:

$\mathrm{C} \mathrm{H}_{0.67} \mathrm{O}_{0.022} \mathrm{~N}_{0.0116} \mathrm{~S}_{0.008}+\mathrm{a} \mathrm{H} \mathrm{H}_{2} \mathrm{O}+\mathrm{b} \mathrm{O} \mathrm{O}_{2}=\mathrm{e} \mathrm{CO}+\mathrm{f} \mathrm{H}_{2}+\mathrm{g} \mathrm{COS}$

$+\mathrm{j} \mathrm{H}_{2} \mathrm{~S}+\mathrm{kCO}_{2}+1 \mathrm{CH}_{4}+\mathrm{m} \mathrm{H}_{2} \mathrm{O}$

- Reactions in gasifier:

1) Pyrolysis and devolatilization for $\left[\begin{array}{llll}\mathrm{C} & \mathrm{H}_{0.67} & \mathrm{O}_{0.022} & \mathrm{~N}_{0.0116}\end{array}\right.$ $\left.\mathrm{S}_{0.008}\right]$

$\mathrm{C}_{(\mathrm{S})}=0.3345 \mathrm{H}_{2}+0.272 \mathrm{H}_{2} \mathrm{O}+0.0058 \mathrm{~N}_{2}+0.008 \mathrm{H}_{2} \mathrm{~S}+0.0115 \mathrm{O}_{2}$

$\begin{array}{llllll}14.869 & 0.669 & 0.4896 & 0.1624 & 0.272 & 0.3568\end{array}$

$\begin{array}{llllll}2500 T P D & 112.5 & 82.5 & 27.5 & 47.812 & 60\end{array}$

$\mathrm{H}_{2}+\mathrm{S}=\mathrm{H}_{2} \mathrm{~S}$

$2 \quad 32 \quad 34$

$\begin{array}{lll}2.812 & 45 & 47.812\end{array}$

2) Gasification

$\begin{array}{lccc}3 \mathrm{C}_{(\mathrm{S})} & \mathrm{H}_{2} \mathrm{O} \\ 44.607 & 18 & \mathrm{O}_{2}= & 3 \mathrm{CO}\end{array}-\underset{32}{\mathrm{H}_{2}}$

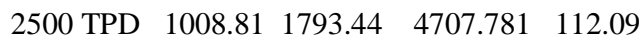

3) Combustion

$\mathrm{CO}+0.5 \mathrm{O}_{2}=\mathrm{CO}_{2}$

$\begin{array}{lll}28 & 16 & 44\end{array}$

536.48 TPD $306.56 \quad 843.04$

4) Water from partial combustion

$\mathrm{H}_{2}+0.5 \mathrm{O}_{2}=\mathrm{H}_{2} \mathrm{O}$ (Water with slag)

$2 \quad 16 \quad 18$

$\begin{array}{lll}7.5 & 60 \quad 67.5\end{array}$

- Mass of gasses in syngas $\dot{\mathrm{m} C O} 2=843.04$ TPD $\dot{\mathrm{m}} \mathrm{CO}=4171.301 \mathrm{TPD}$

$\dot{\mathrm{m}} \mathrm{H}_{2}=214.278 \mathrm{TPD} \dot{\mathrm{m}}_{\mathrm{N} 2}=27.5 \mathrm{TPD}$

$\dot{\mathrm{m}}_{\mathrm{H} 2 \mathrm{~S}}=47.812 \mathrm{TPD} \dot{\mathrm{m}}_{\mathrm{steam}}=82.5 \mathrm{TPD}$

Mass of syngas $(\dot{\mathrm{m}} \operatorname{syn}(1))=843.04+4171.301+214.278+27.5+$

$47.812+82.5$ TPD

$\dot{\mathrm{m}}_{\mathrm{Syn}(1)}=5386.431 \mathrm{TPD}$

- Cold gas efficiency

$\mathrm{LHV}$ of $\mathrm{H}_{2}=121000 \mathrm{~kJ} / \mathrm{kg}$

$\mathrm{LHV}$ of $\mathrm{CO}=10095 \mathrm{~kJ} / \mathrm{kg}$

$\mathrm{LHV}$ of $\mathrm{CH}_{4}=49995 \mathrm{~kJ} / \mathrm{kg} \quad \mathrm{HHV}_{\mathrm{COAL}}=33.3 \mathrm{MJ} / \mathrm{kg}$

$\Delta \mathrm{HC}_{\mathrm{C}}=300088.4+487375.97=787464.37 \mathrm{~kW}$
$-\mathrm{CGE}=[\Delta \mathrm{HC} /(\dot{\mathrm{m}} \times \mathrm{HHV})$ Coal $] \times 100=$ $[787464.37 / 963541.67] \times 100=81.726 \%$

- Carbon conversion

$\mathrm{CCE}=\dot{\mathrm{m}}_{\mathrm{C}}$ in coal $/ \dot{\mathrm{m}}_{\mathrm{C}}$ in syngas $=(2500 / 2500) \times 100=100 \%$

- Syngas cooler

The syngas cooler cools the syngas by the boiler fire tubes which are heated by radiation and convective heat transfer. Assume the heat by radiation is equal to the heat needed to change water in slurry to vapor for gasification.

Summation of heat in syngas cooler, $\mathrm{S}=\left[\mathrm{CCE} \times \mathrm{HHV}_{\mathrm{Coal}}\right]-\Delta \mathrm{H}_{\mathrm{C}}$ $\mathrm{S}=963541.67-787464.37=176077.3 \mathrm{~kW}$

This heat converts water to superheated steam

Steam: $\mathrm{P}=9 \mathrm{MPa} \mathrm{T}=1150^{\circ} \mathrm{C} \mathrm{h}=4997 \mathrm{~kJ} / \mathrm{kg}$

Water: $\mathrm{P}=9 \mathrm{MPa} \mathrm{T}=25^{\circ} \mathrm{C} \quad \mathrm{h}=105 \mathrm{~kJ} / \mathrm{kg}$

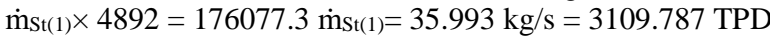

- Gas turbine

Pressure ratio $=15.8$

$\mathrm{h}_{1}=300.19 \mathrm{~kJ} / \mathrm{kg} \mathrm{P}_{\mathrm{r}}=1.386$ for air inlet to the compressor

$\mathrm{h}_{3}=2566.4 \mathrm{~kJ} / \mathrm{kg} \mathrm{P}_{\mathrm{r}}=3464$ for gas inlet to the turbine

1) Combustor

Heat of combustion $\Delta \mathrm{H}_{\mathrm{C}}=787464.37 \mathrm{~kW}$

$\dot{\mathrm{m}}_{\mathrm{Syn}}=\dot{\mathrm{m}}_{\mathrm{Syn}(1)}-\dot{\mathrm{m}}_{\mathrm{H} 2 \mathrm{~S}}-\dot{\mathrm{m}}_{\mathrm{Steam}}=5386.431-47.812-82.5=$

$5256.119 \mathrm{TPD}=60.835 \mathrm{~kg} / \mathrm{s}$

$-\mathrm{H}_{2}+0.5\left(\mathrm{O}_{2}+3.78 \mathrm{~N}_{2}\right)=\mathrm{H}_{2} \mathrm{O}+1.89 \mathrm{~N}_{2}$ $\begin{array}{llll}2 & 68.92 & 18 & 52.92\end{array}$

214.278 TPD $7384.02 \quad 1928.502 \quad 5669.796$

$-\mathrm{CO}+0.5\left(\mathrm{O}_{2}+3.78 \mathrm{~N}_{2}\right)=\mathrm{CO}_{2}+1.89 \mathrm{~N}_{2}$

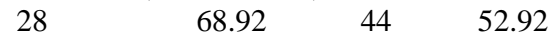

4171.301 TPD $\quad 10267.359 \quad 6554.902 \quad 7883.759$

For complete combustion mair $=17651.379 \mathrm{TPD}=204.298 \mathrm{~kg} / \mathrm{s}$

$-\mathrm{O}_{2}+3.78 \mathrm{~N}_{2}=$ air $32 \quad 105.84 \quad 137.84$

2100 TPD $\quad 6945.75 \quad 9045.75$

$\dot{\mathrm{m}}_{\mathrm{N} 2}=6945.75 \mathrm{TPD}=80.39 \mathrm{~kg} / \mathrm{s}$

$\Delta \mathrm{H}_{\mathrm{C}}=\mathrm{h}_{3}\left(\dot{\mathrm{m}}_{\mathrm{air}}+\dot{\mathrm{m}}_{\mathrm{N} 2}+\dot{\mathrm{m}}_{\mathrm{Syn}}\right)-\mathrm{h}_{2} \dot{\mathrm{m}}_{\text {air }}$

$787464.37=2566.4\left(\dot{\mathrm{m}}_{\mathrm{air}}+80.39+60.835\right)-660.16 \dot{\mathrm{m}}_{\text {air }}$

$425024.53=1906.24 \dot{\mathrm{m}}_{\mathrm{air}}$

$\dot{\mathrm{m}}_{\mathrm{air}}=222.965 \mathrm{~kg} / \mathrm{s} \dot{\mathrm{m}}_{\mathrm{gas}}=364.19 \mathrm{~kg} / \mathrm{s}$

2) Air compressor

The pressure ratio $=15.8$ and the air inlet conditions are $\mathrm{T}=300 \mathrm{~K}$, $\mathrm{P}=1$ bar, $\mathrm{h}=300.19 \mathrm{~kJ} / \mathrm{kg}, \mathrm{Pr}=1.386$

The outlet conditions are $\mathrm{T}=650 \mathrm{~K}, \mathrm{P}=15.8 \mathrm{bar}, \mathrm{h}=660.16 \mathrm{~kJ} / \mathrm{kg}$, $\operatorname{Pr}=21.898$

$\dot{\mathrm{m}}_{\text {air }}=222.965 \mathrm{~kg} / \mathrm{s}$

$\dot{\mathrm{m}}_{\mathrm{gas}}=\dot{\mathrm{m}}_{\mathrm{air}}+\dot{\mathrm{m}}_{\mathrm{N} 2}+\dot{\mathrm{m}}_{\mathrm{Syn}}=364.19 \mathrm{~kg} / \mathrm{s}$

Power of compressor $=\dot{m}_{\text {air }}\left(h_{o}-h_{\text {in }}\right)=222.965(660.16-300.19)$

$\mathrm{P}_{\mathrm{C}}=80260.711 \mathrm{~kW}$

3) Gas turbine 
We find the outlet conditions from the gas turbine

For pressure ratio $=15.8$, the outlet conditions are: $\operatorname{Pr}=219.24, \mathrm{~T}=$ $1175 \mathrm{~K}$

$\mathrm{P}=1 \mathrm{bar}, \mathrm{h}=1249.68 \mathrm{~kJ} / \mathrm{kg}$

Power of turbine $\mathrm{P}_{\mathrm{T}}=\dot{\mathrm{m}}_{\text {gas }} \times\left(\mathrm{h}_{\mathrm{o}}-\mathrm{h}_{\text {in }}\right)=479536.26 \mathrm{~kW}$

Power of gas turbine $=\mathrm{P}_{\mathrm{T}}-\mathrm{P}_{\mathrm{C}}=399275.55 \mathrm{~kW}$

$\eta_{C}=\left[w_{\text {net }} / \Delta H_{C}\right] \times 100=50.704 \%$

- Heat recovery gas system (HRGS)

1) inlet:

a) Water at $\mathrm{T}_{\mathrm{a}}=25^{\circ} \mathrm{C}, \mathrm{P}_{\mathrm{a}}=9 \mathrm{MPa}, \mathrm{h}_{\mathrm{a}}=105 \mathrm{~kJ} / \mathrm{kg}$

b) Gas at $\mathrm{T}_{\mathrm{G} 1}=1175 \mathrm{~K}, \mathrm{~h}_{\mathrm{Gl}}=1249.68 \mathrm{~kJ} / \mathrm{kg}, \dot{\mathrm{m}}_{\mathrm{Gl}}=364.19 \mathrm{~kg} / \mathrm{s}$

2) outlet:

a) Steam at $\mathrm{T}_{b}=1150{ }^{\circ} \mathrm{C}, \mathrm{Pb}=9 \mathrm{MPa}, \mathrm{h}_{b}=4997 \mathrm{~kJ} / \mathrm{kg}$

b) $\mathrm{Gas}$ at $\mathrm{T}_{\mathrm{G} 2}=370 \mathrm{~K}, \mathrm{~h}_{\mathrm{G} 2}=370.67 \mathrm{~kJ} / \mathrm{kg}$

$\dot{\mathrm{m}}_{\mathrm{a}} \mathrm{h}_{\mathrm{a}}+\dot{\mathrm{m}}_{\mathrm{G} 1} \mathrm{~h}_{\mathrm{G} 1}=\dot{\mathrm{m}}_{\mathrm{st}} \mathrm{h}_{\mathrm{st}}+\dot{\mathrm{m}}_{\mathrm{G} 2} \mathrm{~h}_{\mathrm{G} 2}$

$\dot{\mathrm{m}}_{\mathrm{st}}=320126.65 / 4892=65.439 \mathrm{~kg} / \mathrm{s}=5653.929 \mathrm{TPD}$

- Steam turbine

1) Inlet conditions

$\dot{\mathrm{m}}_{\mathrm{st}}$ in $=101.432 \mathrm{~kg} / \mathrm{s}, \mathrm{T}=1150{ }^{\circ} \mathrm{C}, \mathrm{P}=9 \mathrm{MPa}$,

$\mathrm{h}=4997 \mathrm{~kJ} / \mathrm{kg}, \mathrm{s}=8.1648 \mathrm{~kJ} / \mathrm{kg} \mathrm{K}$

2) Outlet conditions

$\mathrm{T}=45^{\circ} \mathrm{C}, \mathrm{P}=0.1 \mathrm{bar}, \mathrm{X}=1, \mathrm{~h}=2584.7 \mathrm{~kJ} / \mathrm{kg}, \mathrm{s}=8.1648 \mathrm{~kJ} / \mathrm{kg} \mathrm{K}$

Power of turbine $=\dot{m}_{s t} \times\left(h_{\text {in }}-h_{0}\right)=244684.41 \mathrm{~kW}$

- Condenser

The cooling water cools steam in condenser from saturated steam $X=1$ to saturated water $X=0$, this water cooling is coming from a cooling tower where the inlet temperature $=50{ }^{\circ} \mathrm{C}$ and the outle temperature $=25{ }^{\circ} \mathrm{C}$. Thus, the mass flow rate of cooling water, $\dot{m}_{w}$ is estimated from the energy balance equation $\dot{\mathrm{m}}_{\mathrm{st}}\left(\mathrm{h}_{\mathrm{in}}-\mathrm{h}_{\mathrm{o}}\right)=\dot{\mathrm{m}}_{\mathrm{w}}\left(\mathrm{h}_{\mathrm{o}}-\mathrm{h}_{\mathrm{in}}\right)$

$101.432 \times(2584.7-105)=\dot{\mathrm{m}}_{\mathrm{w}} \times 4.18 \times(50-25)$

Mass of cooling water $=2406.899 \mathrm{~kg} / \mathrm{s}=207956.06 \mathrm{TPD}$

- Oxygen blown in gasifier (main compressor)

Pressure in gasifier $=30 \mathrm{bar}$

1) Inlet conditions: $\mathrm{T}=300 \mathrm{~K}, \mathrm{P}=1 \mathrm{bar}, \mathrm{h}=300.19 \mathrm{~kJ} / \mathrm{kg}$

2) Outlet conditions: $\mathrm{T}=770 \mathrm{~K}, \mathrm{P}=30 \mathrm{bar}, \mathrm{h}=789.11 \mathrm{~kJ} / \mathrm{kg}$ $\dot{\mathrm{m}}_{\text {air }}=9045.75 \mathrm{TPD}=104.696 \mathrm{~kg} / \mathrm{s}$ $\mathrm{P}_{\mathrm{C}}=\dot{\mathrm{m}}_{\text {air }}\left(\mathrm{h}_{\mathrm{o}}-\mathrm{h}_{\text {in }}\right)=51187.968 \mathrm{~kW}$

- Cycle efficiency
Total power $=399275.55+244684.41-51187.968=592772 \mathrm{~kW}$

$\eta_{\text {th }}=\left[\mathrm{P}_{\text {Total }} / \dot{\mathrm{m}}_{\text {Coal }} \mathrm{HHV}\right] \times 100=61.52 \%$

- $\mathrm{CO}_{2}$ emission

Mass of $\mathrm{CO}_{2}$ emission $=7397.942 \mathrm{TPD}$

$\mathrm{CO}_{2}$ emission $\%=100 \%$

- Economics

a) The capital cost per unit power output $=1145 \$ / \mathrm{kW}$

Capital cost $=\$ 678.706$ million

Output power $=592257.56 \mathrm{~kW}$

b) The cost of electricity (COE) per unit energy output $(\$ / \mathrm{kWh})$.

The COE is calculated over the entire useable life of the plant.

The entire useable life of the plant $=20$ years

Capital cost $=\$ 678.706$ million

Operating costs $=$ ??

- Fuel $=$ Bituminous Coal

Tone $=\$ 47$

$\dot{\mathrm{m}}_{\mathrm{Coal}}=2500 \mathrm{TPD}$

Fuel cost $=2500 \times 47 \times 365=42887500 \$ /$ year

- Operation and maintenance cost $=2 \%$ of the capital cost $/$ year

$\mathrm{O} \& \mathrm{M}=0.02 \times 678.706=13.574 \$ \mathrm{M} /$ year

So operating costs $=56.461 \$$ year

For 20 years the costs $=678.706+(56.461 \times 20)=\$ 1807.926$ million

Total cost in first year $\$$ million $=\$ 735.167$ million

Unit output energy $=(592257.56 \times 8760) / 1000=5188176.2$

MWh

Cost of electricity $(\mathrm{COE})$ per unit energy output $=141.7 \$ / \mathrm{MW}$

\subsection{Energy balance and economics of steam power plant with CCS (Post combustion).}

The representative steam power plant, shown in Fig. 3 comprises a boiler, heater tubes, boiler drum, carbon capture system, steam turbines, generator, cooling tower, pumps, valves and condenser. The output of this station is $393 \mathrm{MW}$ and its thermal efficiency is $40.8 \%$ with a capital cost of $\$ 816$ million.

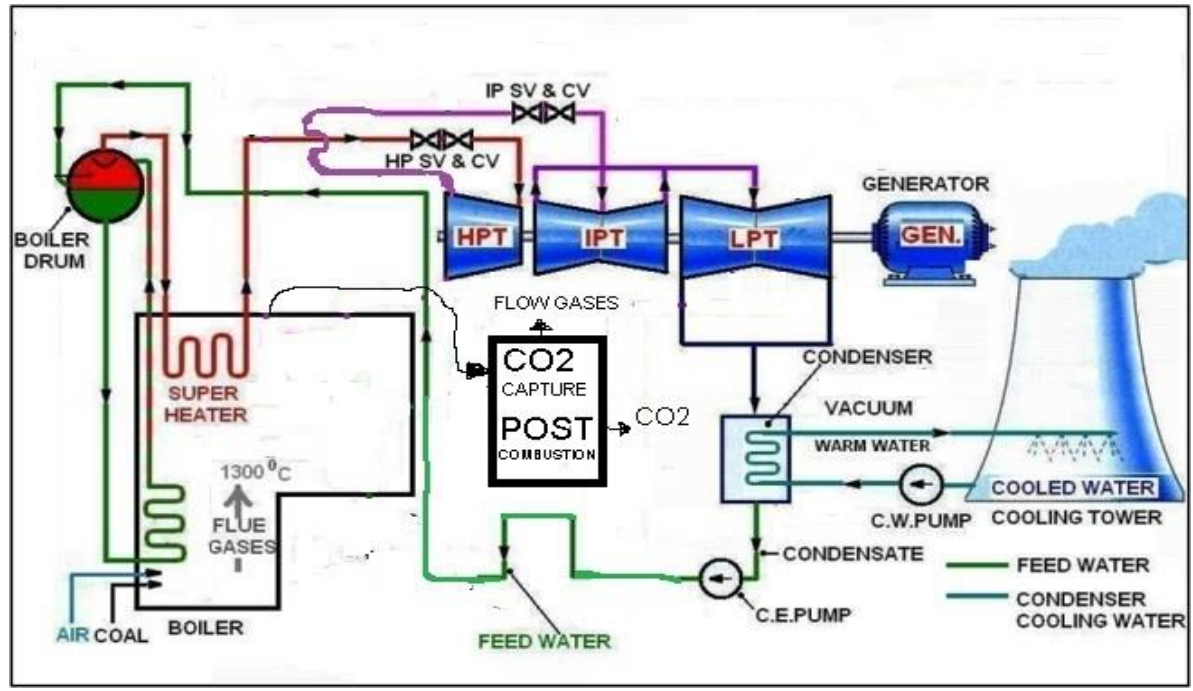

Fig. 3: Steam Power Plant with CCS [3].

- Properties of coal

Coal type: Bituminous coal

Coal composition: $\mathrm{C}_{0.67} \mathrm{O}_{0.022} \mathrm{~N}_{0.0116} \mathrm{~S}_{0.008}$

$\mathrm{M}_{\mathrm{C}}=100 / 6.725=14.869 \mathrm{gm} / \mathrm{mole}$

Moisture $=3.3 \%$ of weight Ash $=6.2 \%$ of weight

$\dot{\mathrm{m}}_{\mathrm{C}}=2500 \mathrm{TPD} \mathrm{HHV}=33.3 \mathrm{MJ} / \mathrm{kg}$

- Boiler

- Chemical reaction in boiler
$\mathrm{C} \mathrm{H}_{0.67} \mathrm{O}_{0.022} \mathrm{~N}_{0.0116} \mathrm{~S}_{0.008 .}+1.1645\left(\mathrm{O}_{2}+3.78 \mathrm{~N}_{2}\right)=\mathrm{CO}_{2}+0.335$ $\mathrm{H}_{2} \mathrm{O}+0.008 \mathrm{SO}_{2}+4.40181 \mathrm{~N}_{2}$

$\begin{array}{llllll}14.869 & 160.51468 & 44 & 6.03 & 0.512 & 123.25068\end{array}$

2500TPD 26988.143 $7397.5 \quad 1013.8543 \quad 86.085144 \quad 20722.759$

For complete combustion $\dot{\mathrm{m}}_{\mathrm{a}}=26988.143 \mathrm{TPD}=312.363 \mathrm{~kg} / \mathrm{s}$

- Calculations of boiler

Summation of heat in boiler, $\mathrm{S}=[\dot{\mathrm{m}} \times \mathrm{HHV}] \mathrm{c}$

$\mathrm{S}=963541.67 \mathrm{~kW}$

This heat converts water to superheated steam 
Steam: $\mathrm{P}=9 \mathrm{MPa}, \mathrm{T}=1150^{\circ} \mathrm{C}, \mathrm{h}=4997 \mathrm{~kJ} / \mathrm{kg}$

Water: $\mathrm{P}=9 \mathrm{MPa}, \mathrm{T}=25{ }^{\circ} \mathrm{C}, \mathrm{h}=105 \mathrm{~kJ} / \mathrm{kg}$

$\dot{\mathrm{m}}_{\mathrm{St}} \times 4892=963541.67 \dot{\mathrm{m}} \mathrm{St}=196.962 \mathrm{~kg} / \mathrm{s}=17017.517 \mathrm{TPD}$

- Steam turbine

1) Inlet conditions

$\dot{\mathrm{m}}_{\mathrm{st}}$ in $=196.962 \mathrm{~kg} / \mathrm{s}, \mathrm{T}=1150{ }^{\circ} \mathrm{C}, \mathrm{P}=9 \mathrm{MPa}$,

$\mathrm{h}=4997 \mathrm{~kJ} / \mathrm{kg}, \mathrm{s}=8.1648 \mathrm{~kJ} / \mathrm{kg} \mathrm{K}$

2) Outlet conditions

$\mathrm{T}=45^{\circ} \mathrm{C}, \mathrm{P}=0.1 \mathrm{bar}, \mathrm{X}=1, \mathrm{~h}=2584.7 \mathrm{~kJ} / \mathrm{kg}, \mathrm{s}=8.1648 \mathrm{~kJ} / \mathrm{kg} \mathrm{K}$

Power of turbine $=\dot{\mathrm{m}}_{\mathrm{st}} \times\left(\mathrm{h}_{\text {in }}-\mathrm{h}_{\mathrm{o}}\right)=475131.43 \mathrm{~kW}$

- Condenser

The cooling water in the condenser converts steam from saturated $X=1$ to saturated water $X=0$, this cooling water is coming fed from a cooling tower where the inlet and outlet temperatures are 50 and $25{ }^{\circ} \mathrm{C}$, respectively. Now the mass flow rate of cooling water, $\dot{\mathrm{m}}_{\mathrm{w}}$ can be obtained from the energy balance equation $\dot{\mathrm{m}}_{\mathrm{st}} \times\left(\mathrm{h}_{\mathrm{in}}-\mathrm{h}_{\mathrm{o}}\right)=\dot{\mathrm{m}}_{\mathrm{w}} \times\left(\mathrm{h}_{\mathrm{o}}-\mathrm{h}_{\text {in }}\right)$

$196.962 \times(2584.7-105)=\dot{\mathrm{m}}_{\mathrm{w}} \times 4.18 \times(50-25)$

Mass of cooling water $=4673.7481 \mathrm{~kg} / \mathrm{s}=403811.83 \mathrm{TPD}$

- $\mathrm{CO}_{2}$ compressor of carbon capture ( post combustion)

1) Inlet conditions are: $\mathrm{CO}_{2}$ at $\mathrm{P}=1 \mathrm{bar}, \mathrm{h}=214.34 \mathrm{~kJ} / \mathrm{kg}$

2) Outlet conditions are: $\mathrm{P}=150 \mathrm{bar}, \mathrm{h}=1172.772 \mathrm{~kJ} / \mathrm{kg}$ $\dot{\mathrm{m}}_{\mathrm{CO} 2}=7397.5 \mathrm{TPD}=85.624 \mathrm{~kg} / \mathrm{s}$

$\mathrm{P}_{\mathrm{C}}=\dot{\mathrm{m}}_{\mathrm{CO} 2}\left(\mathrm{~h}_{\mathrm{o}}-\mathrm{h}_{\text {in }}\right)=85.624 \times(1172.772-214.34)=82064.782$ $\mathrm{kW}$

- Cycle efficiency

1) For low pressure $\mathrm{CO}_{2}$

Total power $=475131.43 \mathrm{~kW}$

$n_{\text {th }}=\left[\mathrm{P}_{\text {Total }} / \dot{\text { m}}_{\text {coal }} \mathrm{HHV}\right] \times 100=49.31 \%$

2) For high pressure $\mathrm{CO} 2$

Total power $=475131.43-82064.782=393066.65 \mathrm{~kW}$

$n_{\text {th }}=\left[\mathrm{P}_{\text {Total }} / \dot{m}_{\text {coal }}\right.$ HHV $] \times 100=40.794 \%$

- $\mathrm{CO}_{2}$ emission

$\dot{\mathrm{m}}_{\mathrm{CO} 2}$ capture $=7397.942 \mathrm{TPD}$

Mass of CO2 emission $=7397.942-7397.942=0$ TPD

$\mathrm{CO}_{2}$ capture $\%=100 \%$

$\mathrm{CO}_{2}$ emission $\%=0 \%$

- Economics

- Power plant with LP CCS

a) The capital cost per unit power output $=1718 \$ / \mathrm{kW}$

Capital cost $=\$ 816.275$ million

Output Power $=475131.43 \mathrm{~kW}$

b) The cost of electricity (COE) per unit energy output ( $\$ / \mathrm{kWh})$.

The COE is calculated over the entire useable life of the plant.

The entire useable life of the plant $=20$ years

Capital cost $=\$ 816.275$ million

Operating costs $=? ?$

- $\quad$ Fuel $=$ Bituminous Coal

Tone $=\$ 47$

$\dot{\mathrm{m}}_{\text {Coal }}=2500 \mathrm{TPD}$

Fuel cost $=2500 \times 47 \times 365=42887500 \$ /$ year

- Operation and maintenance cost $=2 \%$ of the capital cost / year

$\mathrm{O} \& \mathrm{M}=0.02 \times 816.275=16.325 \$ \mathrm{M} /$ year

So the operating costs $=59.213 \$ \mathrm{M} /$ year

For 20 years the costs $=816.275+(59.213 \times 20)=\$ 2000.535$ million
Unit output energy $=(475131.43 \times 8760) / 1000=4162151.3$ MWh

Total cost in first year $\$$ million $=\$ 875.488$ million

Cost of electricity (COE) per unit energy output $=210.345$ $\$ / \mathrm{MWh}$

b) The cost of $\mathrm{CO}_{2}$ capture.

Cost of $\mathrm{CO}_{2}$ captured (or removed) $=$

(COE $\mathrm{CCS}_{-}$- $\left.\mathrm{CE}_{\mathrm{NonCCS}}\right) \$ / \mathrm{MWh} /\left(\mathrm{CO}_{2}\right.$ captured) Ton/MWh m. $\mathrm{CO}_{2}$ Capatur $=7397.5$ TPD

$\mathrm{CO}_{2}$ captured $(\mathrm{Ton} / \mathrm{MWh})=(7397.5 \times 365) / 4162151.3=0.648$ Tone/MWh

Cost of $\mathrm{CO}_{2}$ captured $=(210.345-137.804) / 0.648=111.946$

$\$ /$ Tone

- Power plant with HP CCS

a) Capital cost per unit power output $=1718 \$ / \mathrm{kW}$

Capital cost $=\$ 816.275$ million

Output power $=475131.43 \mathrm{~kW}$

b) The cost of electricity (COE) per unit energy output $(\$ / \mathrm{kWh})$.

We calculate the COE over the entire useable life of the plant.

Entire useable life of the plant $=20$ years

Capital cost $=\$ 816.275$ million

The operating costs $=$ ??

- $\quad$ Fuel $=$ Bituminous Coal

Tone $=\$ 47$

$\dot{\mathrm{m}} \mathrm{Coal}=2500 \mathrm{TPD}$

The fuel cost $=2500 \times 47 \times 365=42887500 \$$ /year

- Operation and maintenance cost $=2 \%$ of the capital cost $/$ year

$\mathrm{O} \& \mathrm{M}=0.02 \times 816.275=16.325 \$ \mathrm{M} /$ year

So the operating costs $=59.213 \$ \mathrm{M} /$ year

For 20 years the costs $=816.275+(59.213 \times 20)=\$ 2000.535$ million

Unit output energy $=(393066.65 \times 8760) / 1000=3443263.9$

MWh

Total cost in first year $\$$ million $=\$ 875.488$ million

Cost of electricity (COE) per unit energy output $=254.261$ $\$ / \mathrm{MWh}$

c) The cost of $\mathrm{CO} 2$ capture.

Cost of $\mathrm{CO}_{2}$ captured (or removed) $=$

( $\left.\mathrm{COE}_{\mathrm{CCS}}-\mathrm{COE}_{\mathrm{NonCCS}}\right) \$ / \mathrm{MWh} /\left(\mathrm{CO}_{2}\right.$ captured $) \mathrm{Ton} / \mathrm{MWh}$

$\dot{\mathrm{m}}_{\mathrm{CO} 2 \text { capture }}=7397.5 \mathrm{TPD}$

$\mathrm{CO}_{2}$ captured $(\mathrm{Ton} / \mathrm{MWh})=(7397.5 \times 365) / 3443263.9=0.784$

Tone/MWh

Cost of $\mathrm{CO}_{2}$ captured $=(254.261-137.804) / 0.784=148.542$

\$/Tone

\subsection{Energy balance and economics of entrained flow gasification plant with CCS (pre combustion)}

Figure 4 shows the selected entrained flow gasification plant which includes the gasifier, heater tubes, syngas cooler, gas cleanup, air separation unit, slurry plant, carbon capture system, gas turbine, steam turbine, generators, cooling tower, pumps, valves, and condenser. The plant produces $481.5 \mathrm{MW}$ with $50 \%$ efficiency with a capital cost $\$ 791$ million. 


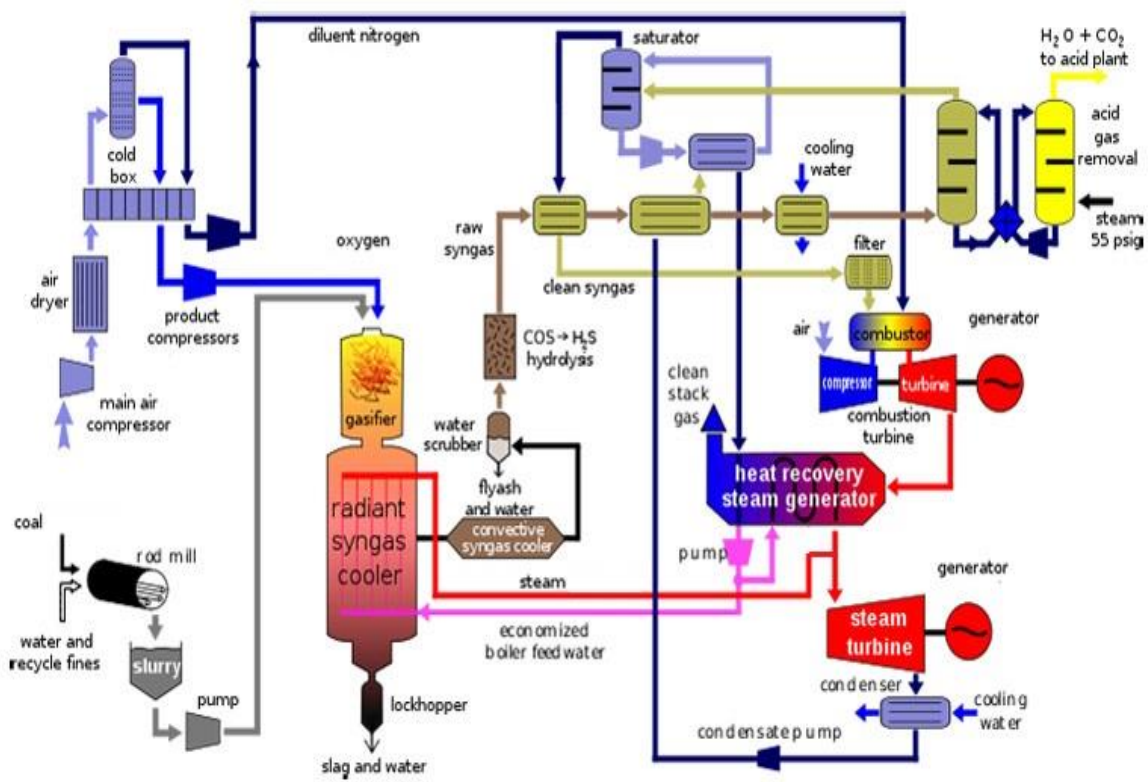

Fig. 4: Entrained Flow Gasification Plant with CCS [3].

- $\quad$ Properties of coal

Coal type: Bituminous coal

Coal composition: $\mathrm{C} \mathrm{H}_{0.67} \mathrm{O}_{0.022} \mathrm{~N}_{0.0116} \mathrm{~S}_{0.008}$

$\mathrm{M}_{\mathrm{C}}=100 / 6.725=14.869 \mathrm{gm} / \mathrm{mole}$

Moisture $=3.3 \%$ of weight

Ash $=6.2 \%$ of weight

micoal $=2500$ TPD

$\dot{\mathrm{m}} 2=2100$ TPD $84 \%$ by weight of coal

$\dot{\mathrm{m}}_{\mathrm{H} 20}=1008.81 \mathrm{TPD} 40.352 \%$ of [slurry feed]

Moisture $=82.5$ TPD

Ash $=155$ TPD

- Gasifier

Chemical reaction in gasifier:

$\mathrm{C} \mathrm{H}_{0.67} \mathrm{O}_{0.022} \mathrm{~N}_{0.0116} \mathrm{~S}_{0.008}+\mathrm{a} \mathrm{H}_{2} \mathrm{O}+\mathrm{b} \mathrm{O}_{2}=\mathrm{e} \mathrm{CO}+\mathrm{f} \mathrm{H}_{2}+\mathrm{g} \mathrm{COS}$

$+\mathrm{j} \mathrm{H}_{2} \mathrm{~S}+\mathrm{k} \mathrm{CO}_{2}+1 \mathrm{CH}_{4}+\mathrm{m} \mathrm{H}_{2} \mathrm{O}$

- Reactions in gasifier:

1) Pyrolysis and devolatilization for $\left[\mathrm{C} \mathrm{H} \mathrm{H}_{0.67} \mathrm{O}_{0.022} \mathrm{~N}_{0.0116} \mathrm{~S}_{0.008}\right.$ ]

$\mathrm{C}_{(\mathrm{S})}=0.3345 \mathrm{H}_{2}+0272 \mathrm{H}_{2} \mathrm{O}+0.0058 \mathrm{~N}_{2}+0.008 \mathrm{H}_{2} \mathrm{~S}+0.0115 \mathrm{O}_{2}$

$\begin{array}{llllll}14.869 & 0.669 & 0.4896 & 0.1624 & 0.272 & 0.3568\end{array}$

$\begin{array}{lllll}2500 T P D & 112.5 & 82.5 & 27.5 & 47.812\end{array}$

$\mathrm{H}_{2}+\mathrm{S}=\mathrm{H}_{2} \mathrm{~S}$

$2 \quad 32 \quad 34$

$2.812 \quad 45 \quad 47.812$

2) Gasification

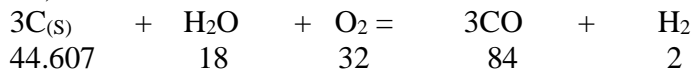

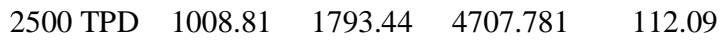

3) Combustion

$\mathrm{CO}+0.5 \mathrm{O}_{2}=\mathrm{CO}_{2}$

$\begin{array}{lll}28 & 16 & 44\end{array}$

536.48 TPD $\quad 306.56 \quad 843.04$

4) Water from partial combustion

$\mathrm{H}_{2}+0.5 \mathrm{O}_{2}=\mathrm{H}_{2} \mathrm{O}$ (Water with slag)

$2 \quad 16 \quad 18$

$\begin{array}{lll}7.5 & 60 & 67.5\end{array}$

- Mass of gasses in syngas

$\dot{\mathrm{m}}_{\mathrm{CO} 2}=843.04$ TPD $\dot{\mathrm{m}}_{\mathrm{CO}}=4171.301 \mathrm{TPD}$

$\dot{\mathrm{m}}_{\mathrm{H} 2}=214.278 \mathrm{TPD} \dot{\mathrm{m}}_{\mathrm{N} 2}=27.5 \mathrm{TPD}$

$\dot{\mathrm{m}} \mathrm{H} 2 \mathrm{~S}=47.812$ TPD $\dot{\mathrm{m}} \mathrm{steam}=82.5 \mathrm{TPD}$

Mass of syngas $=843.04+4171.301+214.278+27.5+47.812+$

82.5

$\dot{\mathrm{m}}_{\mathrm{Syn}(1)}=5386.431 \mathrm{TPD}$

- Cold gas efficiency

$\mathrm{LHV}$ of $\mathrm{H}_{2}=121000 \mathrm{~kJ} / \mathrm{kg}$

$\mathrm{LHV}$ of $\mathrm{CO}=10095 \mathrm{~kJ} / \mathrm{kg}$
$\mathrm{LHV}$ of $\mathrm{CH}_{4}=49995 \mathrm{~kJ} / \mathrm{kg} \mathrm{HHV}_{\text {Coal }}=33.3 \mathrm{MJ} / \mathrm{kg}$

$\Delta \mathrm{H}_{\mathrm{C}}=300088.4+487375.97=787464.37 \mathrm{~kW}$

$\mathrm{CGE}=\left[\Delta \mathrm{H}_{\mathrm{C}} /(\dot{\mathrm{m}} \times \mathrm{HHV})_{\mathrm{Coal}}\right] \times 100=[787464.37 /$

$963541.67] \times 100$

$\mathrm{CGE}=81.726 \%$

- Carbon conversion

$\mathrm{CCE}=\dot{\mathrm{m}}$ Carbon in coal $/ \dot{\mathrm{m}}$ Carbonin syngas $=(2500 / 2500) \times 100=$ $100 \%$

- Syngas cooler

The syngas cooler cools the syngas from 1480 to $316^{\circ} \mathrm{C}$ by means of the boiler fire tubes which are heated by radiation and convective heat transfer.

Summation of heat of reaction, $\mathrm{S}=\left[\mathrm{CGE} \times \mathrm{HHV}_{\mathrm{Coal}}\right]-\Delta \mathrm{H}_{\mathrm{C}}$

$\mathrm{S}=963541.67-787464.37=176077.3 \mathrm{~kW}$

This heat converts water to superheated steam

Steam: $\mathrm{P}=9 \mathrm{MPa} \mathrm{T}=1150^{\circ} \mathrm{C} \mathrm{h}=4997 \mathrm{~kJ} / \mathrm{kg}$

Water: $\mathrm{P}=9 \mathrm{MPa} \mathrm{T}=25^{\circ} \mathrm{C} \mathrm{h}=105 \mathrm{~kJ} / \mathrm{kg}$

$\dot{\mathrm{m} S t}(1) \times 4892=176077.3$

$\dot{\mathrm{m} S t}(1)=35.993 \mathrm{~kg} / \mathrm{s}=3109.787 \mathrm{TPD}$

- Carbon capture (pre combustion)

- Reaction in CCS (pre combustion)

$\mathrm{CO}+\mathrm{H}_{2} \mathrm{O}=\mathrm{CO}_{2}+\mathrm{H}_{2}$

$\begin{array}{llll}28 & 18 & 44 & 2\end{array}$

$\begin{array}{llll}4171.301 & 2681.551 & 6554.902 & 297.95\end{array}$

$\dot{\mathrm{m}}_{\mathrm{H} 2}=512.228 \mathrm{TPD}=5.928 \mathrm{~kg} / \mathrm{s}$

$\dot{\mathrm{m}}_{\mathrm{H} 2 \mathrm{O}}=2681.551-82.5 \mathrm{TPD}=2599.051 \mathrm{TPD}=30.081 \mathrm{~kg} / \mathrm{s}$

$\dot{\mathrm{m}}_{\mathrm{Syn}}=512.228+27.5=539.728 \mathrm{TPD}=6.247 \mathrm{~kg} / \mathrm{s}$

- Heat in CCS (Pre combustion)

$\Delta \mathrm{HW}_{\text {with } \mathrm{CCS}}=717356.34 \mathrm{~kW}$

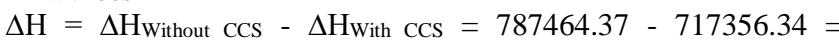
$70108.03 \mathrm{~kW}$

1) Inlet:

$\dot{\mathrm{m}}_{\text {Water }}=31.036 \mathrm{~kg} / \mathrm{s}, \mathrm{h}_{\text {in }}=105 \mathrm{~kJ} / \mathrm{kg}, \mathrm{P}=380 \mathrm{kPa}$

2) outlet:

$\Delta \mathrm{H}=\dot{\mathrm{m}}_{\text {Water }}\left(\mathrm{ho}-\mathrm{h}_{\text {in }}\right) \mathrm{h}_{\mathrm{o}}=2258.926 \mathrm{~kJ} / \mathrm{kg}$

- Gas turbine

Pressure ratio $=15.8$

$\mathrm{h}_{1}=300.19 \mathrm{~kJ} / \mathrm{kg} \mathrm{P}_{\mathrm{r}}=1.386$ for air inlet to the compressor

$\mathrm{h}_{3}=2566.4 \mathrm{~kJ} / \mathrm{kg} \mathrm{P}_{\mathrm{r}}=3464$ for gas inlet to the turbine

1) Combustor

Heat of combustion $\Delta \mathrm{H}_{\mathrm{C}}=717356.34 \mathrm{kw}$

$\dot{\mathrm{m}}_{\text {Syn }}=6.247 \mathrm{~kg} / \mathrm{s}$

$-\mathrm{H}_{2}+0.5\left(\mathrm{O}_{2}+3.78 \mathrm{~N}_{2}\right)=\mathrm{H}_{2} \mathrm{O}+1.89 \mathrm{~N}_{2}$

$\begin{array}{llll}2 & 68.92 & 18 & 52.92\end{array}$

512.228 TPD $\quad 17651.377 \quad 4610.052 \quad 13553.553$

For complete combustion $\dot{\mathrm{m}}_{\mathrm{air}}=17651.377 \mathrm{TPD}=204.3 \mathrm{~kg} / \mathrm{s}$ 


$$
\begin{aligned}
& -\quad \mathrm{O}_{2}+3.78 \mathrm{~N}_{2}=\quad \text { air } \\
& 32 \quad 105.84 \quad 137.84 \\
& 2100 \mathrm{TPD} \quad 6945.75 \quad 9045.75 \\
& \dot{\mathrm{m}}_{\mathrm{N} 2}=6945.75 \mathrm{TPD}=80.39 \mathrm{~kg} / \mathrm{s} \\
& \Delta \mathrm{HC}_{\mathrm{C}}=\mathrm{h}_{3}\left(\dot{\mathrm{m}}_{\text {air }}+\dot{\mathrm{m}}_{\mathrm{N} 2}+\dot{\mathrm{m}}_{\mathrm{Syn}}\right)-\mathrm{h}_{2} \dot{\mathrm{m}}_{\text {air }} \\
& 717356.34=2566.4\left(\dot{\mathrm{m}}_{\text {air }}+80.39+6.247\right)-660.16 \dot{\mathrm{m}}_{\text {air }} \\
& 495011.14=1906.24 \dot{\mathrm{m}}_{\text {air }} \\
& \dot{\mathrm{m}}_{\text {air }}=259.679 \mathrm{~kg} / \mathrm{s} \dot{\mathrm{m}}_{\text {gas }}=346.316 \mathrm{~kg} / \mathrm{s} \\
& \text { 2) Air compressor }
\end{aligned}
$$

Pressure ratio $=15.8$ and the inlet air conditions are $\mathrm{T}=300 \mathrm{~K}, \mathrm{P}=$ $1 \mathrm{bar}, \mathrm{h}=300.19 \mathrm{~kJ} / \mathrm{kg}, \operatorname{Pr}=1.386$

The outlet conditions are $\mathrm{T}=650 \mathrm{~K}, \mathrm{P}=15.8 \mathrm{bar}, \mathrm{h}=660.16 \mathrm{~kJ} / \mathrm{kg}$, $\operatorname{Pr}=21.898$

$\dot{\mathrm{m}}_{\text {air }}=259.679 \mathrm{~kg} / \mathrm{s}$

$\dot{\mathrm{m}}_{\mathrm{gas}}=\dot{\mathrm{m}}_{\mathrm{air}}+\dot{\mathrm{m}}_{\mathrm{N} 2}+\dot{\mathrm{m}}_{\mathrm{Syn}}=346.316 \mathrm{~kg} / \mathrm{s}$

Power of compressor $=\dot{m}_{\text {air }}\left(h_{o}-h_{\text {in }}\right)=259.679(660.16-300.19)$

$\mathrm{P}_{\mathrm{C}}=93476.65 \mathrm{~kW}$

3) Gas turbine

We find the outlet conditions from the gas turbine

For pressure ratio $=15.8$, the outlet conditions are: $\operatorname{Pr}=219.24, \mathrm{~T}=$ $1175 \mathrm{~K}$,

$\mathrm{P}=1 \mathrm{bar}, \mathrm{h}=1249.68 \mathrm{~kJ} / \mathrm{kg}$

Power of turbine $\mathrm{P}_{\mathrm{T}}=\dot{\mathrm{m}}_{\mathrm{gas}} \times\left(\mathrm{h}_{\mathrm{o}}-\mathrm{h}_{\text {in }}\right)=456001.2 \mathrm{~kW}$

Power of gas turbine $=\mathrm{P}_{\mathrm{T}}-\mathrm{P}_{\mathrm{C}}=362524.55 \mathrm{~kW}$

$\eta_{\mathrm{C}}=\left[\mathrm{w}_{\text {net }} / \Delta \mathrm{H}_{\mathrm{C}}\right] \times 100=50.536 \%$

- Heat recovery gas system (HRGS)

1) inlet:

a) Water at $\mathrm{T}_{\mathrm{a}}=25^{\circ} \mathrm{C}, \mathrm{P}_{\mathrm{a}}=9 \mathrm{MPa}, \mathrm{h}_{\mathrm{a}}=105 \mathrm{~kJ} / \mathrm{kg}$

b) $\mathrm{Gas}$ at $\mathrm{T}_{\mathrm{G} 1}=1175 \mathrm{~K}, \mathrm{~h}_{\mathrm{G} 1}=1249.68 \mathrm{~kJ} / \mathrm{kg}, \dot{\mathrm{m}}_{\mathrm{G} 1}=346.316 \mathrm{~kg} / \mathrm{s}$

c) Steam (mixture) out let of CCS, h= $2363.926 \mathrm{~kJ} / \mathrm{kg}, \dot{\mathrm{m}}_{\mathrm{st}}=$ $30.081 \mathrm{~kg} / \mathrm{s}$

2) outlet:

a) Steam at $\mathrm{T}_{b}=1150{ }^{\circ} \mathrm{C}, \mathrm{P}_{\mathrm{b}}=9 \mathrm{MPa}, \mathrm{h}_{\mathrm{b}}=4997 \mathrm{~kJ} / \mathrm{kg}$

b) Gas at $\mathrm{T}_{\mathrm{G} 2}=370 \mathrm{~K}, \mathrm{~h}_{\mathrm{G} 2}=370.67 \mathrm{~kJ} / \mathrm{kg}$

c) Steam (saturated vapor), $\mathrm{h}=2736.2 \mathrm{~kJ} / \mathrm{kg}$

$\dot{\mathrm{m}}_{\mathrm{a}} \mathrm{h}_{\mathrm{a}}+\dot{\mathrm{m}}_{\mathrm{G} 1} \mathrm{~h}_{\mathrm{G} 1}+\left(\dot{\mathrm{m}}_{\mathrm{st}} \mathrm{h}\right)_{\text {in }}=\dot{\mathrm{m}}_{\mathrm{st}} \mathrm{h}_{\mathrm{st}}+\dot{\mathrm{m}}_{\mathrm{G} 2} \mathrm{~h}_{\mathrm{G} 2}+\left(\dot{\mathrm{m}}_{\mathrm{st}} \mathrm{h}\right)_{\text {out }}$

$\dot{\mathrm{m}}_{\mathrm{st}}=(304415.23-11198.374) / 4892=59.938 \mathrm{~kg} / \mathrm{s}=5178.643$ TPD

- Steam turbine

1) Inlet conditions

$\dot{\mathrm{m}}_{\mathrm{st}}$ in $=95.931 \mathrm{~kg} / \mathrm{s}, \mathrm{T}=1150{ }^{\circ} \mathrm{C}, \mathrm{P}=9 \mathrm{MPa}$,

$\mathrm{h}=4997 \mathrm{~kJ} / \mathrm{kg}, \mathrm{s}=8.1648 \mathrm{~kJ} / \mathrm{kg} \mathrm{K}$

2) Outlet conditions

$\mathrm{T}=45^{\circ} \mathrm{C}, \mathrm{P}=0.1 \mathrm{bar}, \mathrm{X}=1, \mathrm{~h}=2584.7 \mathrm{~kJ} / \mathrm{kg}, \mathrm{s}=8.1648 \mathrm{~kJ} / \mathrm{kg} \mathrm{K}$

Power of turbine $=\dot{\mathrm{m}}_{\mathrm{st}} \times\left(\mathrm{h}_{\mathrm{in}}-\mathrm{h}_{\mathrm{o}}\right)=231414.35 \mathrm{~kW}$

- Condenser

The cooling water in the condenser cools the steam from saturated steam $X=1$ to saturated water $X=0$, this water cooling is supplied from a cooling tower for which the inlet temperature $=50^{\circ} \mathrm{C}$ and the outlet temperature $=25^{\circ} \mathrm{C}$.

Then we get the mass flow rate of water cooling $\dot{m}_{\mathrm{w}}$ from the energy balance equation

$\dot{\mathrm{m}}_{\mathrm{st}}\left(\mathrm{h}_{\mathrm{in}}-\mathrm{h}_{\mathrm{o}}\right)=\dot{\mathrm{m}}_{\mathrm{w}}\left(\mathrm{h}_{\mathrm{o}}-\mathrm{h}_{\text {in }}\right)=95.931 \times(2584.7-105)=\dot{\mathrm{m}}_{\mathrm{w}} \times 4.18 \times$ (50-25)

Mass of cooling water $=2276.364 \mathrm{~kg} / \mathrm{s}=196677.9$ TPD

- Oxygen blown in gasifier (main compressor)

Maximum pressure in gasifier $=30 \mathrm{bar}$

1) Inlet conditions: $\mathrm{T}=300 \mathrm{~K}, \mathrm{P}=1 \mathrm{bar}, \mathrm{h}=300.19 \mathrm{~kJ} / \mathrm{kg}$

2) Outlet conditions: $\mathrm{T}=770 \mathrm{~K}, \mathrm{P}=30 \mathrm{bar}, \mathrm{h}=789.11 \mathrm{~kJ} / \mathrm{kg}$ $\dot{\mathrm{m}}_{\text {air }}=9045.75 \mathrm{TPD}=104.696 \mathrm{~kg} / \mathrm{s}$

$\mathrm{P}_{\mathrm{C}}=\dot{\mathrm{m}}_{\text {air }}\left(\mathrm{h}_{\mathrm{o}}-\mathrm{h}_{\text {in }}\right)=51187.968 \mathrm{~kW}$

- $\mathrm{CO}_{2}$ Compressor

1) Inlet conditions are: $70 \%$ of $\mathrm{CO}_{2}, \mathrm{P}=15.8 \mathrm{bar}, \mathrm{h}=560.772$ $\mathrm{kJ} / \mathrm{kg}$, and $30 \% \mathrm{P}=1 \mathrm{bar}, \mathrm{h}=214.34 \mathrm{~kJ} / \mathrm{kg}$

2) Outlet conditions are: $\mathrm{P}=150 \mathrm{bar}, \mathrm{h}=1172.772 \mathrm{~kJ} / \mathrm{kg}$ $\dot{\mathrm{m}}_{\mathrm{CO} 2}=1008.04+6389.9016=7397.942 \mathrm{TPD}=85.624 \mathrm{~kg} / \mathrm{s}$ $\mathrm{P}_{\mathrm{C}}=\dot{\mathrm{m}}_{\mathrm{CO} 2}\left(\mathrm{~h}_{\mathrm{o}}-\mathrm{h}_{\text {in }}\right)=[(0.7 \times 85.624) \times(1172.772-560.772)]+$ $[(0.3 \times 85.624) \times(1172.772-214.34)]=(59.9368 \times 612)+$ $(25.6872 \times 958.432)=36681.322+24619.434=61300.756 \mathrm{~kW}=$ $61.3 \mathrm{MW}$
- Cycle efficiency

1) For low pressure $\mathrm{CO}_{2}$

Total power $=362524.55+231414.35-51187.968=542750.93$

$\mathrm{kW}$

$\eta_{\text {th }}=\left[\mathrm{P}_{\text {Total }} / \dot{\text { m}}_{\text {Coal }} \mathrm{HHV}\right] \times 100=56.328 \%$

2) For high pressure $\mathrm{CO} 2$

Total power $=362525.39+231414.35-51187.968-61300.756=$ $481450.18 \mathrm{~kW}$

$\eta_{\text {th }}=\left[\mathrm{P}_{\text {Total }} /\right.$ meoal $\left._{\text {mHV }}\right] \times 100=49.966 \%$

- $\mathrm{CO}_{2}$ emission

$\dot{m}_{\mathrm{CO} 2}$ capture $=7397.942 \mathrm{TPD}$

Mass of $\mathrm{CO}_{2}$ emission $=7397.942-7397.942=0 \mathrm{TPD}$

$\mathrm{CO}_{2}$ capture $\%=100 \%$

$\mathrm{CO}_{2}$ emission $\%=0 \%$

- Economics

- Power plant with LP CCS

a) Capital cost per unit power output $=1459 \$ / \mathrm{kW}$

Capital cost $=\$ 791.29$ million

Output power $=542351.33 \mathrm{~kW}$

b) The cost of electricity (COE) per unit energy output $(\$ / \mathrm{kWh})$.

The COE is calculated over the entire useable life of the plant.

The entire useable life of the plant $=20$ years

Capital cost $=\$ 791.29$ million

Operating costs $=$ ??

- Fuel = Bituminous coal

Tone $=\$ 47$

$\dot{\mathrm{m}}_{\mathrm{Coal}}=2500 \mathrm{TPD}$

Fuel cost $=2500 \times 47 \times 365=42887500 \$ /$ year

- Operation and maintenance cost $=2 \%$ of the capital cost $/$ year

O \& $\mathrm{M}=0.02 \times 791.29=15.825 \$ \mathrm{M} /$ year

So the operating costs $=58.713$ \$/year

For 20 years the costs $=791.29+(58.713 \times 20)=\$ 1965.55$ million

Unit output energy $=(542351.33 \times 8760) / 1000=4750997.7$

MWh

Total cost in first year $\$$ million $=\$ 850.003$ million

Cost of electricity $(\mathrm{COE})$ per unit energy output $=178.91 \$ / \mathrm{MWh}$

c) The cost of $\mathrm{CO}_{2}$ capturing.

Cost of $\mathrm{CO}_{2}$ captured (or removed) $=$

(COE $\left.\mathrm{CCS}-\mathrm{COE}_{\text {NonCCS }}\right) \$ / \mathrm{MWh} /\left(\mathrm{CO}_{2}\right.$ captured) Ton/MWh

$\dot{\mathrm{m}}_{\mathrm{CO} 2 \text { capture }}=7397.5 \mathrm{TPD}$

$\mathrm{CO}_{2}$ captured $(\mathrm{Ton} / \mathrm{MWh})=(7397.5 \times 365) / 4750997.7=0.568$

$\mathrm{Ton} / \mathrm{MWh}$

Cost of $\mathrm{CO}_{2}$ captured $=(178.91-141.7) / 0.568=65.51 \$ /$ Ton

d) The cost of $\mathrm{CO}_{2}$ capturing avoided.

Cost of $\mathrm{CO}_{2}$ captured (or removed) $=$

(COE $\mathrm{CCS}_{-\mathrm{COE}}$ PC Non CCS) \$/MWh / ( $\mathrm{CO}_{2}$ captured) Ton/MWh minco2capture $=7397.5$ TPD

$\mathrm{CO}_{2}$ captured $(\mathrm{Ton} / \mathrm{MWh})=(7397.5 \times 365) / 4750997.7=0.568$

Ton/MWh

Cost of $\mathrm{CO}_{2}$ captured $=(178.91-137.804) / 0.568=72.369$ \$/Ton

- Power plant with HP CCS

a) The capital cost per unit power output $=1459 \$ / \mathrm{kW}$

Capital cost $=\$ 791.29$ million

Output power $=542351.33 \mathrm{~kW}$

b) The cost of electricity (COE) per unit energy output $(\$ / \mathrm{kWh})$.

Calculations of the COE are made over the entire useable life of the plant.

The entire useable life of the plant $=20$ years

Capital cost $=\$ 791.29$ million

Operating costs $=$ ??

- $\quad$ Fuel $=$ Bituminous Coal

Tone $=\$ 47$

$\dot{\mathrm{m}}_{\mathrm{Coal}}=2500 \mathrm{TPD}$

Fuel cost $=2500 \times 47 \times 365=42887500 \$ /$ year 
- Operation and maintenance cost $=2 \%$ of the capital cost $/$ year

$\mathrm{O} \& \mathrm{M}=0.02 \times 791.29=15.825 \$ \mathrm{M} /$ year

So the operating costs $=58.713 \$ /$ year

For 20 years the costs $=791.29+(58.713 \times 20)=\$ 1965.55$ mil-

lion

Unit output energy $=(481450.18 \times 8760) / 1000=4217503.6$

MWh

Total cost in first year $\$$ million $=\$ 850.003$ million

Cost of electricity (COE) per unit energy output $=201.541$ \$/MWh

c) The cost of $\mathrm{CO}_{2}$ capturing.

Cost of $\mathrm{CO}_{2}$ captured (or removed) $=$

(COE CCS $_{-}$COE $\left.{ }_{\text {NonCCS }}\right)$ \$/MWh / ( $\mathrm{CO}_{2}$ captured) Ton/MWh

mंco2capture $=7397.5$ TPD

$\mathrm{CO}_{2}$ captured $(\mathrm{Ton} / \mathrm{MWh})=(7397.5 \times 365) / 4217503.6=0.64$

Ton/MWh

Cost of $\mathrm{CO}_{2}$ captured $=(201.541-141.7) / 0.64=93.501$ \$/Tone

d) The cost of $\mathrm{CO} 2$ capturing avoided.

Cost of $\mathrm{CO}_{2}$ captured (or removed) $=$

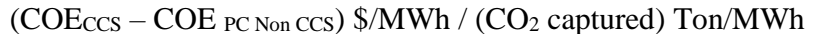

mco2capture $=7397.5$ TPD

$\mathrm{CO}_{2}$ captured $(\mathrm{Ton} / \mathrm{MWh})=(7397.5 \times 365) / 4217503.6$

$=0.64 \mathrm{Ton} / \mathrm{MWh}$

Cost of $\mathrm{CO}_{2}$ captured $=(201.541-137.804) / 0.64$

$=99.589 \$ /$ Tone

\subsection{Comparisons of manual and computer results}

These comparisons are made for the four power stations burning bituminous coal for similar output data. The comparison of results is presented in Tables 3, 4, 5, and 6 .

Table 3: Comparison of Results for Steam Power Plant

\begin{tabular}{lll}
\hline Steam power plant & & \\
\hline Cycle without CCS & Manual results & Computer results \\
Thermal cycle efficiency \% & 49.311 & 49.311 \\
Cost of electricity \$/MWh & 137.804 & 137.804 \\
Cycle with CCS & Manual results & Computer results \\
Thermal cycle efficiency \% & 40.794 & 40.79 \\
Cost of electricity \$/MWh & 254.261 & 254.258 \\
Cost of $\mathrm{CO}_{2}$ capture \$/Tone & 148.542 & 148.509 \\
\hline
\end{tabular}

Table 4: Comparison of Results for Entrained Flow Gasification Power Plant

\begin{tabular}{lll}
\hline \multicolumn{2}{l}{ Entrained flow gasification power plant } & \\
\hline Cycle without CCS & Manual results & Computer results \\
Thermal cycle efficiency \% & 61.52 & 61.52 \\
Cost of electricity \$/MWh & 141.7 & 141.581 \\
Cycle with CCS & Manual results & Computer results \\
Thermal cycle efficiency \% & 49.966 & 50.08 \\
Cost of electricity \$/MWh & 201.541 & 201.612 \\
Cost of $\mathrm{CO}_{2}$ capture \$/Tone & 93.501 & 93.973 \\
\hline
\end{tabular}

Table 5: Comparison of Results for Fluidized Bed Gasification Power Plant

\begin{tabular}{lll}
\hline \multicolumn{2}{l}{ Fluidized bed gasification power plant } & \\
\hline Cycle without CCS & Manual results & Computer results \\
Thermal cycle efficiency \% & 59.416 & 59.42 \\
\hline
\end{tabular}

$\begin{array}{lll}\text { Cost of electricity \$/MWh } & 141.873 & 141.874 \\ \text { Cycle with CCS } & \text { Manual results } & \text { Computer results } \\ \text { Thermal cycle efficiency \% } & 49.701 & 49.70 \\ \text { Cost of electricity \$/MWh } & 197.47 & 197.472 \\ \text { Cost of } \mathrm{CO}_{2} \text { capture \$/Tone } & 95.363 & 95.329\end{array}$

Table 6: Comparison of Results for Fixed Bed Gasification Power Plant Fixed bed gasification power plant

\begin{tabular}{lll} 
Cycle without CCS & Manual results & Computer results \\
Thermal cycle efficiency \% & 62.2 & 62.20 \\
Cost of electricity \$/MWh & 141.49 & 141.491 \\
Cycle with CCS & Manual results & Computer results \\
Thermal cycle efficiency \% & 52.96 & 52.96 \\
Cost of electricity \$/MWh & 192.229 & 192.230 \\
Cost of $\mathrm{CO}_{2}$ capture \$/Tone & 107.726 & 107.566 \\
& & \\
\hline
\end{tabular}

These comparisons indicate indisputable agreement between manual and computer results. This gives unquestionable confidence in our present computer results when used to obtain all the necessary design and comparison data for different coal fired power stations.

\section{Results and discussion}

The objective of this research is to afford a complete thermodynamic design model of power plants which include a steam power plant and an Integrated Gasification Combined Cycle using coal firing. This analysis is conducted by using the present commercial software program so that the detailed thermodynamic data, such as mass flow rate, pressure, temperature, and enthalpy in various places, become available for all interested and beneficiaries.

A comparison is made between power stations with or without carbon capture systems which includes cycle thermal efficiency, cost of electricity, $\mathrm{CO}_{2}$ emission, and cost of $\mathrm{CO}_{2}$ capture. The present comparison is aimed to show which of the power plants studied considered the best according to the discussed items. In the following figures, the power stations are named according to the gasification process used to save space in figures.

\subsection{Thermal cycle efficiency}

The results in this section are presented in two figures. Figure 5 shows the ideal net plant efficiency (LHV) for different gasification processes for all coal ranks. The IGCC plant using the dry-fed fixed bed gasifier has the highest efficiency of $62.2 \%$ (LHV), for all ranks of coal, in comparison with the slurry fed entrained flow gasifiers and dry-fed fluidized bed gasifier. However, it has the lowest throughput of coal fired because of the low temperature since it is a small capacity gasifire type.

Figure 6 depicts the results for the same gasifiers for all coal types but with $\mathrm{CO}_{2}$ capture. The entrained flow gasifier is penalized by a reduction of about $11 \%$ of efficiency by $\mathrm{CO}_{2}$ capture than the dryfed fixed bed processes and the dry-fed fluidized bed processes which only lose $8-9 \%$ of efficiency. The penalty for employing post-combustion carbon capture is about 9-10 \%of efficiency reduction for sub- and super-critical PC plants. A comparison shows that the changes in efficiency of the plants are not much. 


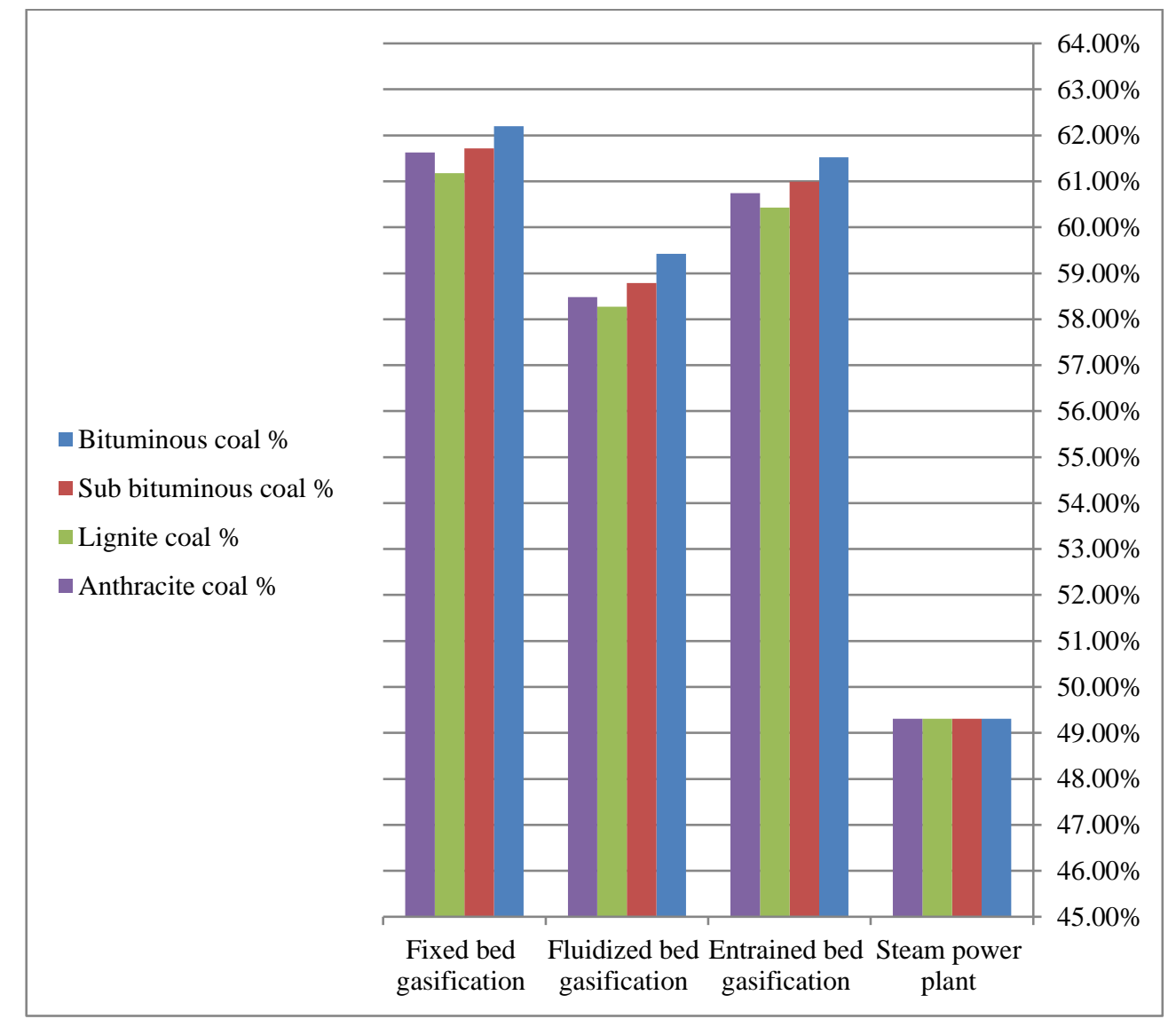

Fig. 5: Thermal Cycle Efficiency without CCS.

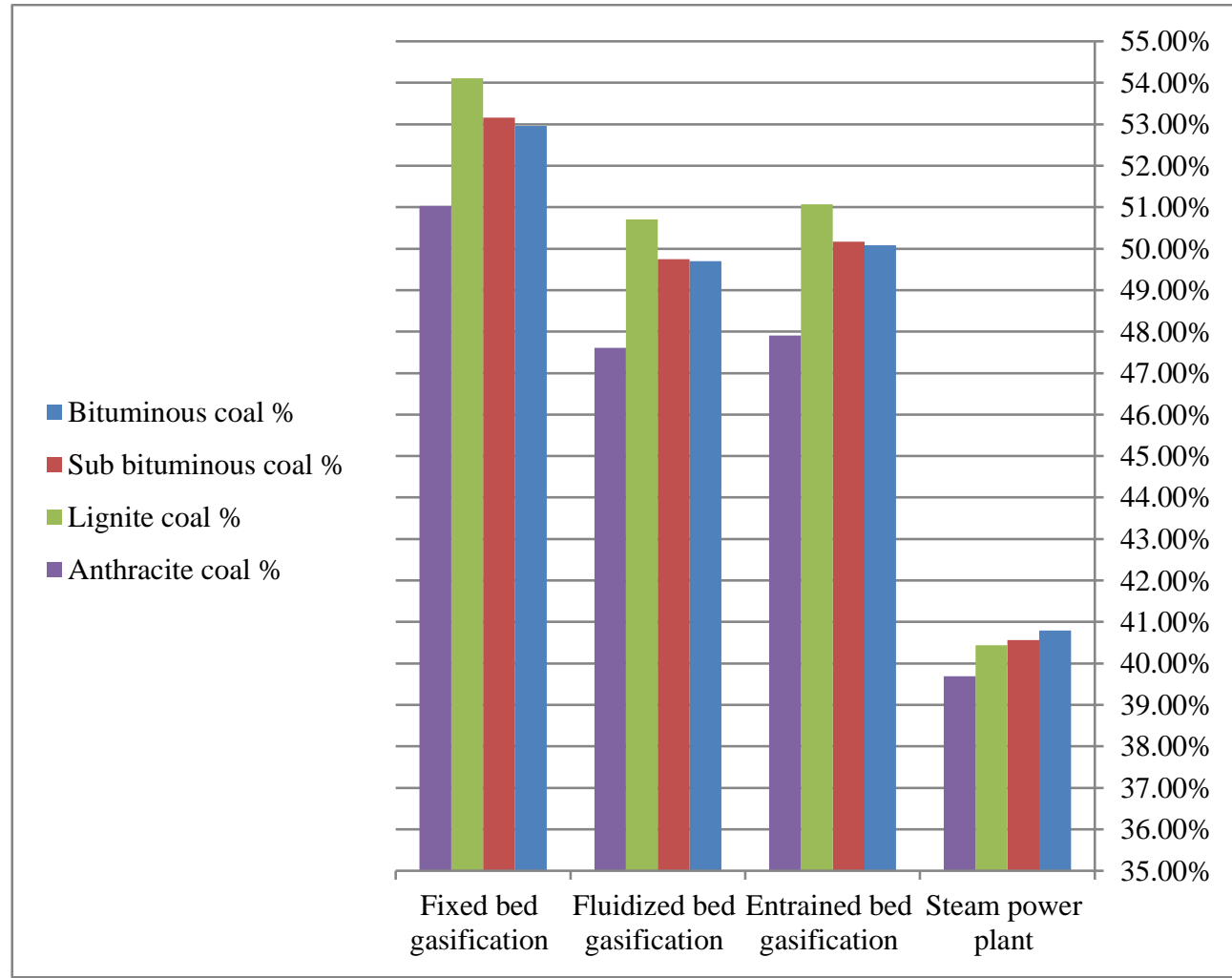

Fig. 6: Thermal Cycle Efficiency with HP CCS.

\subsection{Cost of electricity}

The results here are presented in two figures. Figure 7 represents $\mathrm{COE}$ for several gasification processes for all coal ranks. The trend of COE is different from that of the capital cost in the no- capture condition; the fluidized bed gasification IGCC plant has the highest COE. The PC plant has lowest COEs, but IGCC plants are $2-4 \%$ more expensive. These are for all coal ranks.

Figure 8 shows that when the carbon capture is implemented, the COEs increase by $38-48 \%$ for IGCC plants, and by $80-85 \%$ for PC plants. In IGCC plant the overall cost of electricity (COE) of 
the plant that uses pre combustion carbon capture is slightly cheaper than the PC plants, which use post-combustion carbon capture. The cheaper $\mathrm{COE}$ in case of $\mathrm{CO}_{2}$ capture, for all coals, is obtained for the fixed bed gasifier followed by the fluidized bed. The highest cost is that for the steam power plant.

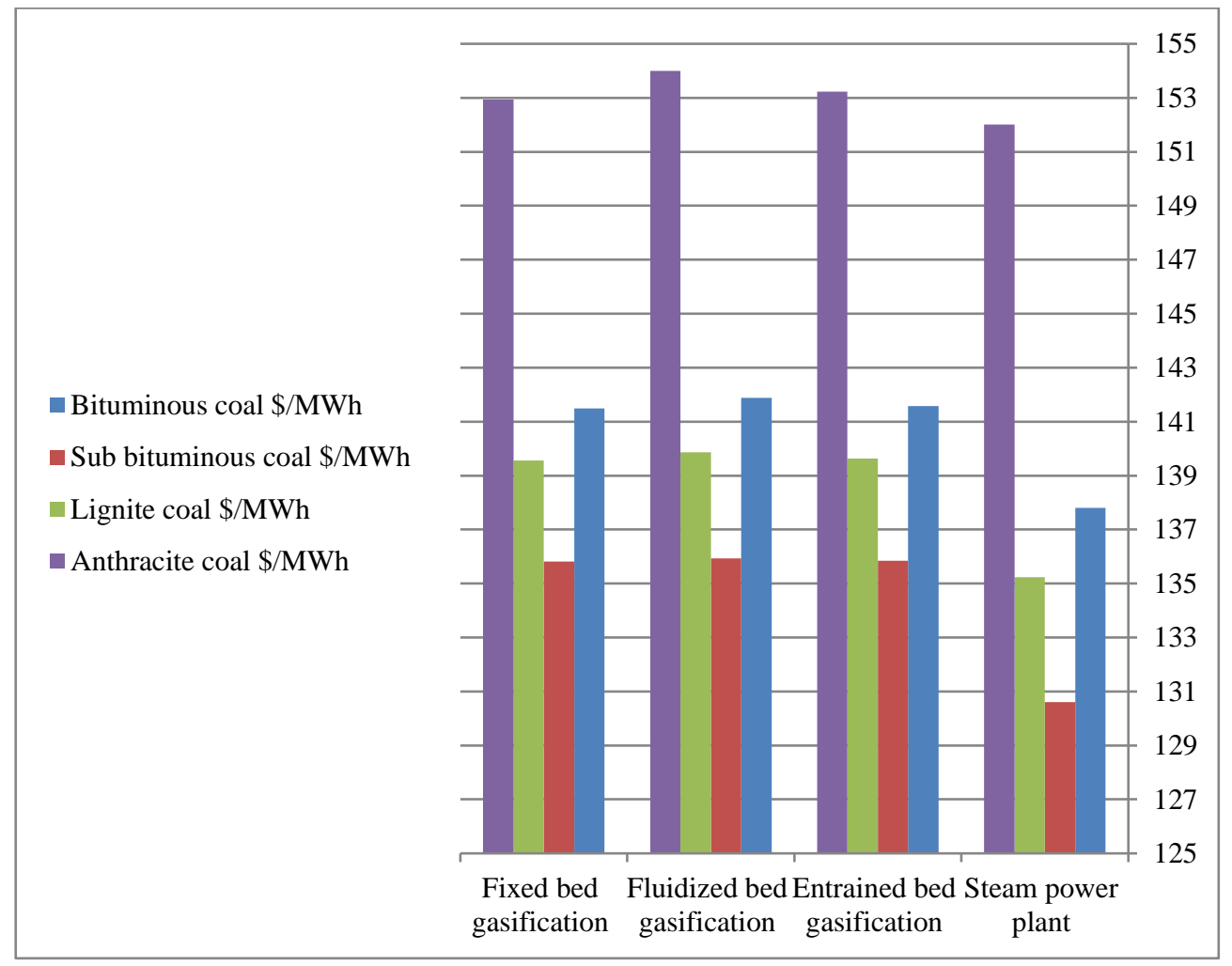

Fig. 7: Cost of Electricity \$/MWh for Plants without CCS.

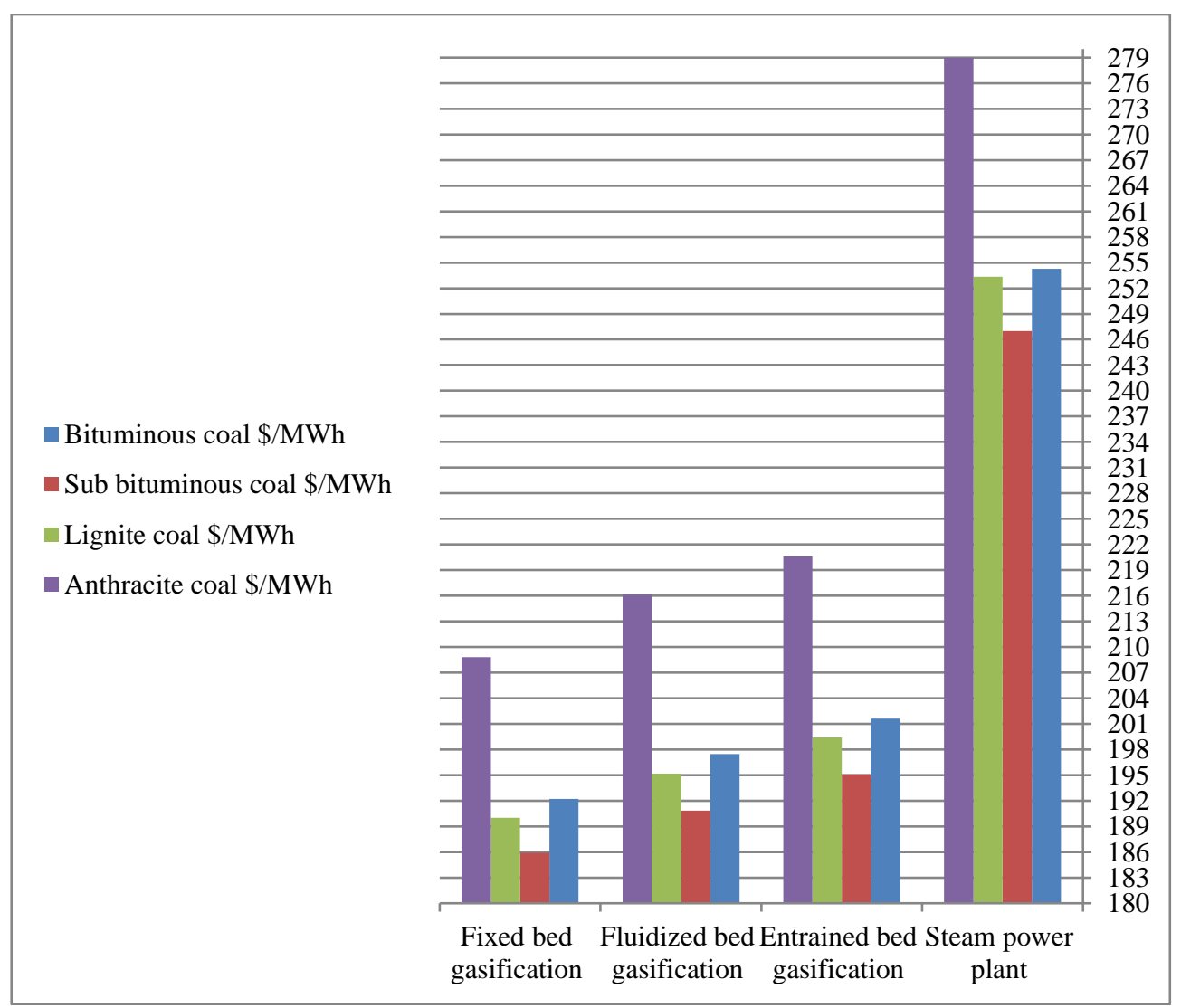

Fig. 8: Cost of Electricity \$/MWh for Plants with HP CCS.

\section{3. $\mathrm{CO}_{2}$ emissions}

Although the COE of IGCC plants are comparable to those of PC plants, it is important to note that the $\mathrm{CO}_{2}$ emissions from the
IGCC plants with carbon capture (as shown in Fig. 9) are significantly lower than those of the PC plants without carbon capture. Therefore, IGCC provides a great opportunity to perform precombustion carbon capture. The $\mathrm{CO}_{2}$ capture cost (not the avoided 
cost of $\mathrm{CO}_{2}$ ) is about 2-3 times cheaper than that for post combustion carbon capture which is used in PC plants.

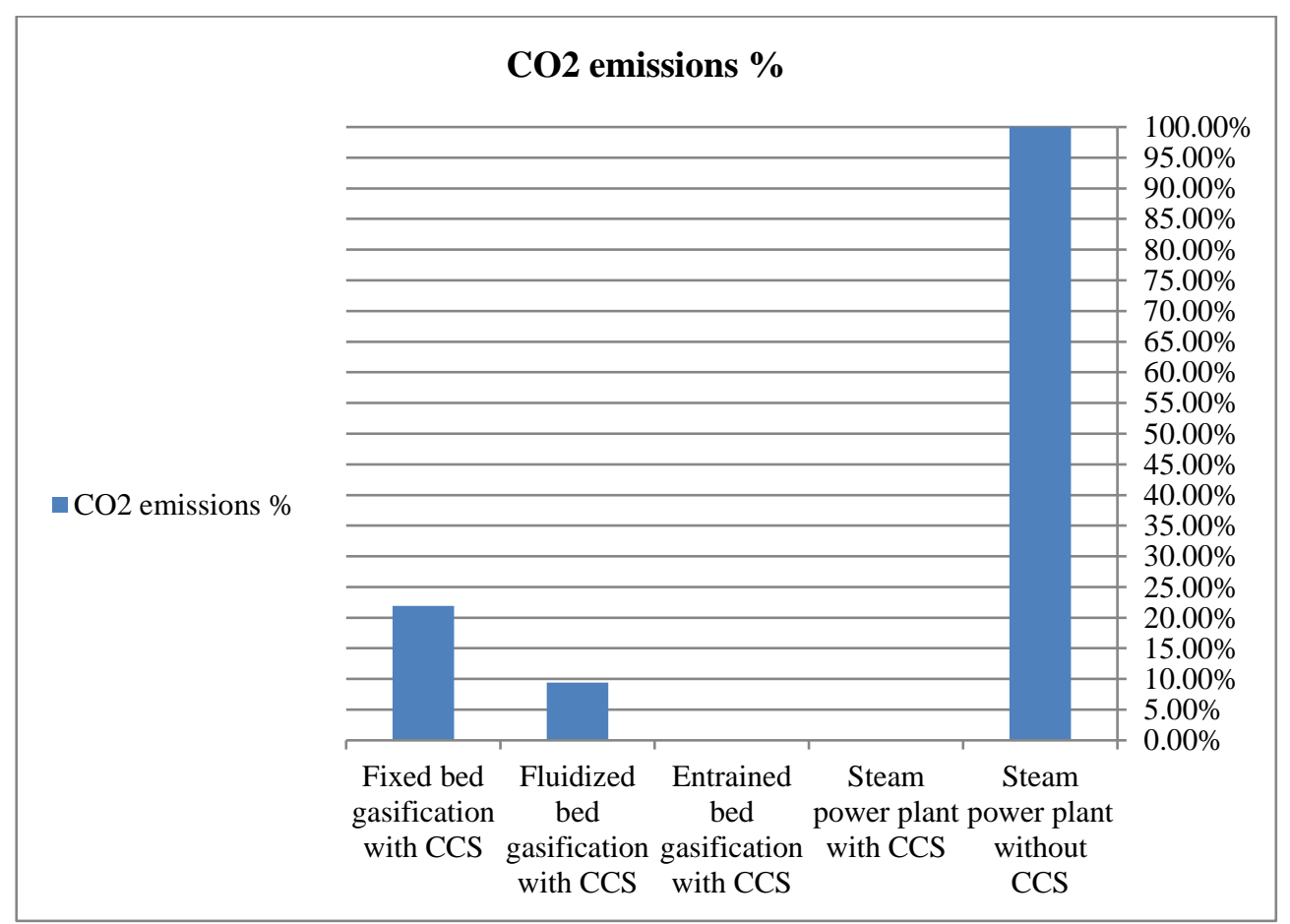

Fig. 9: $\mathrm{CO}_{2}$ Emissions \%.

From these results as offered by the simulation program the entrained flow gasifier and steam power plant, for all coal ranks, are the best because of their low emissions due to the high carbon capture and storage, but the entrained flow gasification power plant with CCS is cheaper than the steam power plant because the latter employs a post combustion carbon capture system.

\subsection{Cost of $\mathrm{CO}_{2}$ capture}

The cost of $\mathrm{CO}_{2}$ captured (or removed) is calculated from the COE difference between analogous plants with and without $\mathrm{CO}_{2}$ capture. The cost of $\mathrm{CO}_{2}$ avoided in reference to a specified plant is the cost which will incentivize carbon capture when a carbon emission tax above this value is levied to both a capture and a defined non-capture reference plant.

Figure 10 depicts the total average costs of $\mathrm{HP} \mathrm{CO}_{2}$ capture for the considered four power plants, for all coal ranks. The results are indicated in Table 7.

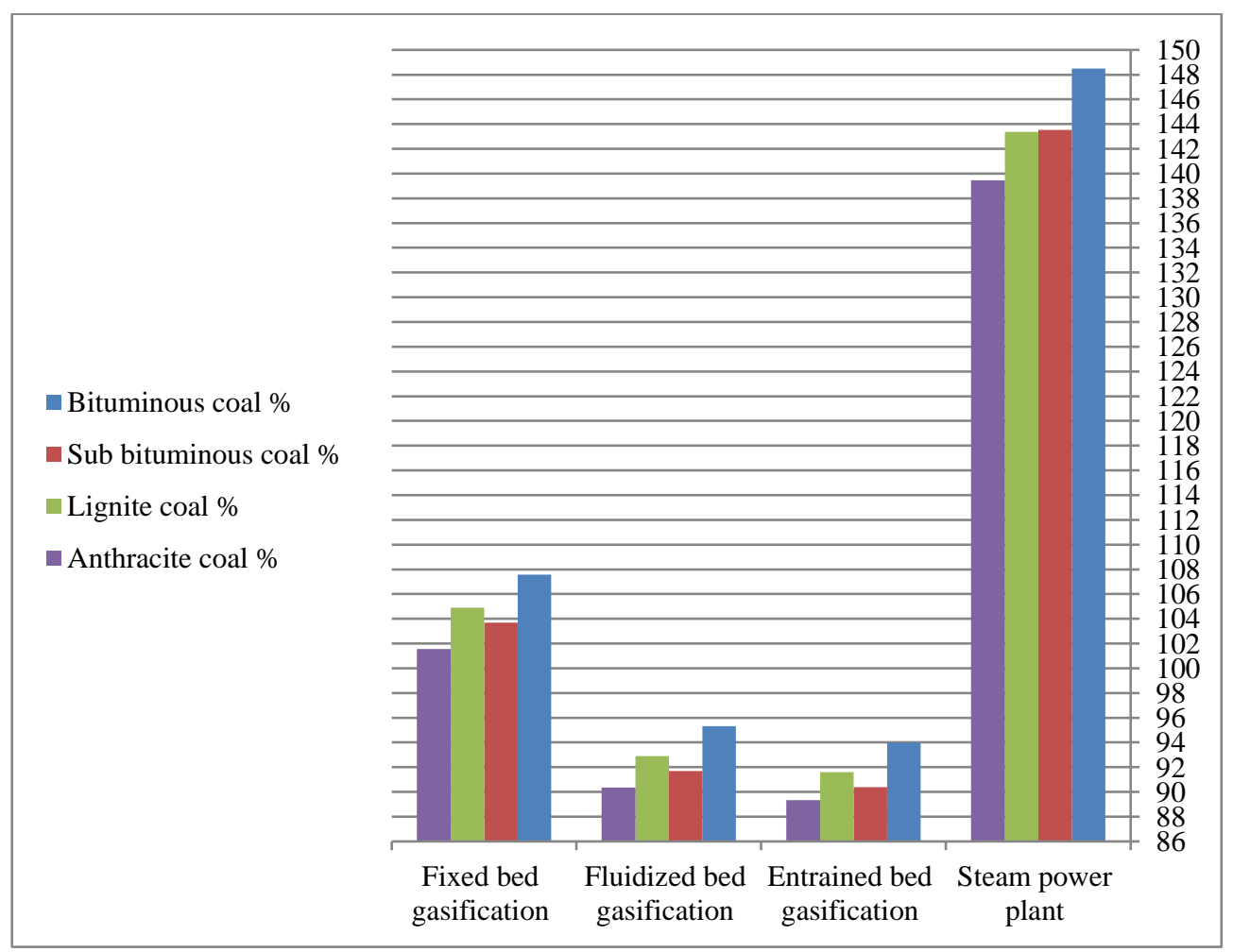

Fig. 10: Cost of HP CO2 Capture \$/Tone. 
Figure 11 gives the total average costs of $\mathrm{HP}^{\mathrm{CO}_{2}}$ capture avoided tabulated in Table 8. for the same above power plants, and coal ranks. The results are

Table 7: Cost of $\mathrm{HP} \mathrm{CO}_{2}$ Capture $\$ /$ Ton

\begin{tabular}{lllll}
\hline \multirow{2}{*}{ Coal rank } & \multicolumn{3}{c}{ Cost $\$$ ton } & Fixed bed Gasification \\
\hline Bituminous coal & Steam power plant & Entrained bed gasification & 95.329 & 107.566 \\
Sub bituminous coal & 148.509 & 93.973 & 91.696 & 103.688 \\
Lignite coal & 143.538 & 90.386 & 92.905 & 104.888 \\
Anthracite coal & 143.376 & 91.602 & 90.344 & 101.56 \\
\hline
\end{tabular}

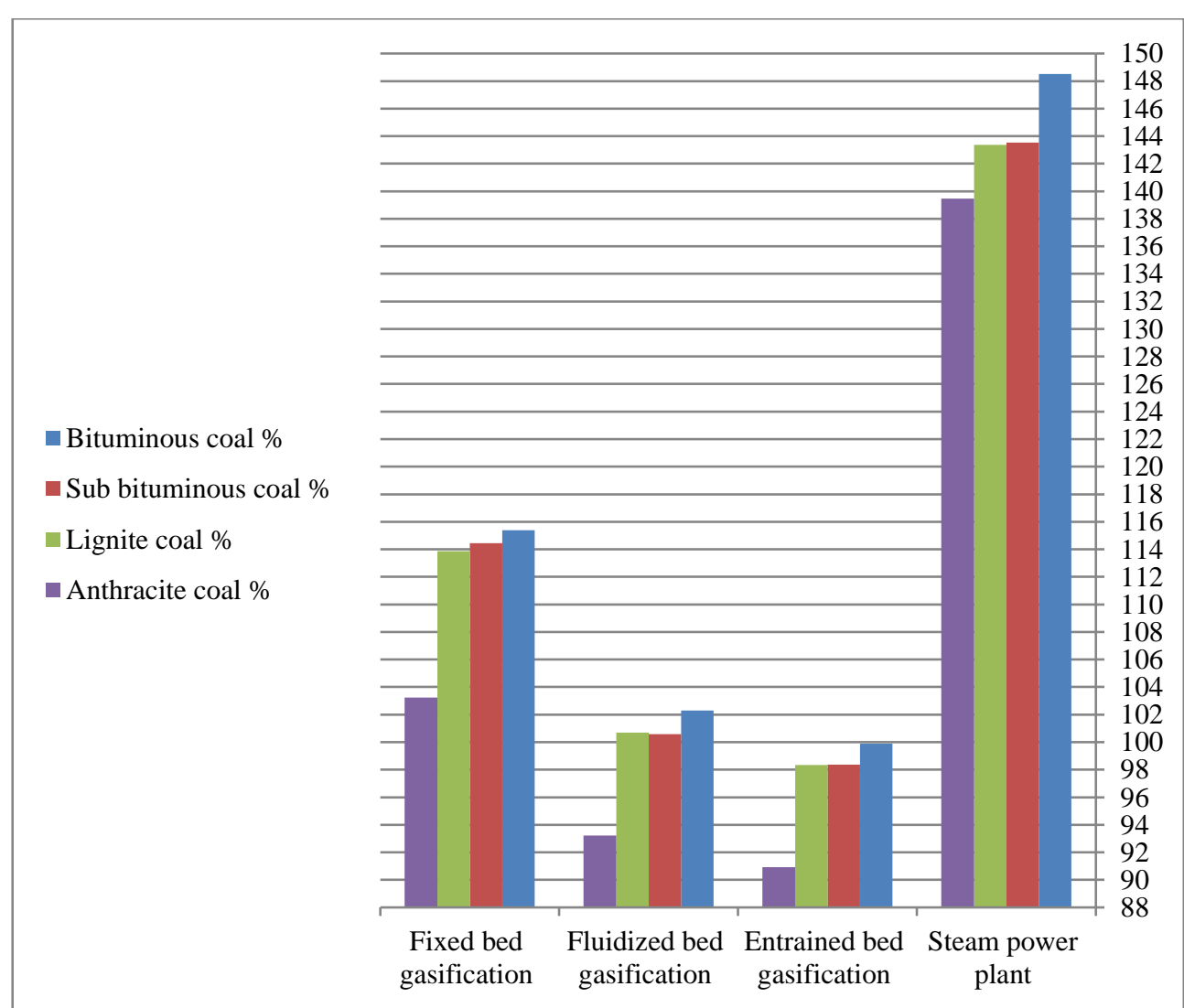

Fig. 11: Cost of $\mathrm{HP} \mathrm{CO}_{2}$ Capture Avoided $\$ /$ Tone.

Table 8: Cost of $\mathrm{HP} \mathrm{CO}_{2}$ Capture Avoided $\$ /$ Ton

\begin{tabular}{lllll}
\hline Coal rank & \multicolumn{3}{c}{ Cost \$/ton } \\
& $\begin{array}{l}\text { Steam power } \\
\text { plant }\end{array}$ & $\begin{array}{l}\text { Entrained bed } \\
\text { Gasification }\end{array}$ & $\begin{array}{l}\text { Fluidized bed } \\
\text { gasification }\end{array}$ & $\begin{array}{l}\text { Fixed bed } \\
\text { gasification }\end{array}$ \\
Bituminous coal & 148.509 & 99.886 & 102.307 & 115.382 \\
Sub bituminous & 143.538 & 98.359 & 100.577 & 114.444 \\
Coal & 143.376 & 98.346 & 100.687 & 113.878 \\
Lignite coal & 139.466 & 90.933 & 93.223 & 103.238 \\
Anthracite coal & & &
\end{tabular}

The results in Figs. 10 and 11 and Tables 7 and 8 indicate that, for all coal types, the entrained bed gasifier plant produced the lowest cost in the two cases, $\mathrm{HP} \mathrm{CO}_{2}$ capture and $\mathrm{HP} \mathrm{CO}_{2}$ capture avoided. This is followed by the fluidized bed gasification technology. The worst in both cases is the steam power plant.

From all the above results furnished by the present simulation program the entrained flow gasification power plant proved to be the most viable one because of its superiority based on all the studied comparison parameters.

\section{Conclusions}

A stupendous achievment of this research is the devolopment of a computer program which provides all thermodynamic, energy, economic data of coal fired power stations. This program is most important for comparing different technologies. The program provides a rather useful versatile powerful tool for designers and operators as well as buyers. It furnishes surplus pithy tangible results.

Comparisons of computer and manual results show excellent agreement, thus our computer program is incridibly reliable and can be used professionally on commercial scale.

The best power plant is the entrained flow gasification power one because of its high thermal efficiency with high throughput of fired coal, low emissions, low cost of carbon capture, however, it is slightly high in cost of electricity because of the high capital cost of the plant.

Finally, IGCC technology offers clear advantages over pulverized coal combustion, especially for achieving higher net efficiency, lower emissions including dust, heavy metals, hazardous compounds, $\mathrm{CO}_{2}$, and gaseous pollutants, and a comparatively lower efficiency penalty for CCS.

\section{Nomenclature}

$\begin{array}{lll}\mathrm{M}_{\mathrm{C}} & \text { Moller weight } & \text { gm/mole } \\ \dot{\mathrm{m}} & \text { Mass flow rate } & \text { Tone per Day (TPD) }\end{array}$




\begin{tabular}{|c|c|c|}
\hline $\mathrm{T}$ & Temperature & ${ }^{0} \mathrm{C}, \mathrm{K}$ \\
\hline $\mathrm{H}$ & Enthalpy & $\mathrm{kJ} / \mathrm{kg}$ \\
\hline HHV & High heating value & $\mathrm{MJ} / \mathrm{kg}$ \\
\hline $\mathrm{P}$ & Pressure, Power & $\mathrm{MPa}, \mathrm{MW}$ \\
\hline S & Summation of heat & $\mathrm{Kw}$ \\
\hline $\mathrm{X}$ & Moisture content & Dimensionless \\
\hline $\mathrm{S}$ & Entropy & $\mathrm{kJ} / \mathrm{kg} \mathrm{K}$ \\
\hline CGE & Cold gas efficiency $\%$ & Dimensionless \\
\hline $\mathrm{CCE}$ & $\begin{array}{l}\text { Carbon conversion } \\
\text { efficiency } \%\end{array}$ & Dimensionless \\
\hline$\Delta \mathrm{H}_{\mathrm{C}}$ & $\begin{array}{l}\text { Heat of combustion of } \\
\text { syngas or combustor }\end{array}$ & MW \\
\hline $\mathrm{P}_{\mathrm{r}}$ & Relative pressure & Dimensionless \\
\hline $\mathrm{O} \& \mathrm{M}$ & $\begin{array}{l}\text { Operating and mainte- } \\
\text { nance cost }\end{array}$ & \$M/year \\
\hline$\eta$ & Efficiency $\%$ & Dimensionless \\
\hline IGCC & Integrated gasificati & combined cycle \\
\hline ASU & Air separation unit & \\
\hline WGS & Water-Gas Shift & \\
\hline CCS & Carbon Capture and & questration \\
\hline $\mathrm{COE}$ & Cost of Electricity & \\
\hline GHG & Greenhouse Gases & \\
\hline $\mathrm{HP}$ & High-Pressure & \\
\hline LP & Low Pressure & \\
\hline HRSG & Heat Recovery Stea & Generator \\
\hline
\end{tabular}

\section{Conflict of interests}

The authors declare that there is no conflict of interests regarding publication of this paper.

\section{References}

[1] Ting Wang, Gary J. Stiegel, Integrated Gasification Combined Cycle (IGCC) Technologies, 1st Edition, Woodhead publishing, Cambridge, England 2016.

[2] http://indianpowersector.com/home/power-station/thermalpower plant/.

[3] http://biofuelsacademy.org/index.html\%3Fp=161.html. 